\title{
Global Existence and Asymptotic Behavior of Solutions for Compressible Two-Fluid Euler-Maxwell Equation
}

\author{
Ismahan Binshati (iD) and Harumi Hattori (D) \\ Department of Mathematics, West Virginia University, Morgantown, WV 26506, USA \\ Correspondence should be addressed to Ismahan Binshati; idbinshati@mix.wvu.edu
}

Received 24 November 2019; Accepted 1 June 2020; Published 29 June 2020

Academic Editor: Wen-Xiu Ma

Copyright ( 2020 Ismahan Binshati and Harumi Hattori. This is an open access article distributed under the Creative Commons Attribution License, which permits unrestricted use, distribution, and reproduction in any medium, provided the original work is properly cited.

We study the global existence and asymptotic behavior of the solutions for two-fluid compressible isentropic Euler-Maxwell equations by the Fourier transform and energy method. We discuss the case when the pressure for two fluids is not identical, and we also add friction between the two fluids. In addition, we discuss the rates of decay of $L^{p}-L^{q}$ norms for a linear system. Moreover, we use the result for $L^{p}-L^{q}$ estimates to prove the decay rates for the nonlinear systems.

\section{Introduction}

We consider the Cauchy problem for the first-order nonlinear two-fluid compressible isentropic Euler-Maxwell equations in three dimensions. In the following system of equations, the first equation is the conservation of the mass. The second equation is conservation of the momentum, to which we added frictional damping $\alpha_{ \pm}\left(u_{ \pm}-u_{\mp}\right)$ besides the damping $v_{ \pm} u_{ \pm}$. Then, the compressible two-fluid Euler-Maxwell equations can be written as

$$
\left\{\begin{array}{l}
\partial_{t} n_{ \pm}+\nabla \cdot\left(n_{ \pm} u_{ \pm}\right)=0 \\
\partial_{t} u_{ \pm}+u_{ \pm} \cdot \nabla u_{ \pm}+\frac{1}{n_{ \pm}} \nabla p_{ \pm}\left(n_{ \pm}\right)= \pm\left(E+u_{ \pm} \times B\right)-v_{ \pm} u_{ \pm}-\alpha_{ \pm}\left(u_{ \pm}-u_{\mp}\right) \\
\partial_{t} E-\nabla \times B=n_{-} u_{-}-n_{+} u_{+} \\
\partial_{t} B+\nabla \times E=0 \\
\nabla . E=n_{+}-n_{-} \\
\nabla \cdot B=0
\end{array}\right.
$$

where $n_{ \pm}=n_{ \pm}(t, x) \in \mathbb{R}^{3}$ denotes the density of electrons $\left(n_{-}\right)$and ions $\left(n_{+}\right), u_{ \pm}=u_{ \pm}(t, x) \in \mathbb{R}^{3}$ denotes the velocity of electrons $\left(u_{-}\right)$and ions $\left(u_{+}\right), E=E(t, x) \in \mathbb{R}^{3}$ denotes the electric field, and $B=B(t, x) \in \mathbb{R}^{3}$ denotes the 
magnetic field for $t>0, x \in \mathbb{R}^{3}$. The initial data are given by

$$
\left.\left[n_{ \pm}, u_{ \pm}, E, B\right]\right|_{t=0}=\left[n_{ \pm 0}, u_{ \pm 0}, E_{0}, B_{0}\right], \quad x \in \mathbb{R}^{3},
$$

with the compatibility conditions

$$
\begin{aligned}
\nabla . E_{0} & =n_{+0}-n_{-0}, \\
\nabla . B_{0} & =0, \\
x & \in \mathbb{R}^{3} .
\end{aligned}
$$

The Euler-Maxwell system (1) is a symmetrizable hyperbolic system for $n>0$, and the initial value problems (1) and (2) have a local smooth solution when the initial data are smooth. The global existence of smooth solutions to the initial boundary value problem has been given in [1] by the compensated compactness method. The authors in $[2,3]$ studied the existence of global smooth solutions for the three-dimensional isentropic Euler-Maxwell system with small amplitude, and the periodic problem was discussed by Uedaet al. [4]. For the special case where the solution to the Euler-Maxwell equation has asymptotic limits with small parameters, see $[5,6]$. The special case of the diffusive relaxation limit of the three-dimensional nonisentropic Euler-Maxwell equation is considered in $[7,8]$. Two hierarchies of models of the ionospheric plasma for twofluid Euler-Maxwell equations were presented in [9]. The Fourier transform method was considered by Duan $[2,10]$ and Kawashima and Ueda [11]. Jerome [12] adapted the classical semigroup-resolvent approach of Kato [13] to the Cauchy problem in $\mathbb{R}^{3}$ and established a local smooth solution. In [2], Duan considered the case when the pressure function $p_{ \pm}(\cdot)$ depend only on density, having the expression $p_{ \pm}\left(n_{ \pm}\right)=A_{ \pm} n_{ \pm}^{\gamma}$ with constants $A_{+}=A_{-}>0$ and the adiabatic exponent $\gamma>1$.

In this paper, we consider the global existence of smooth solutions for the two-fluid compressible isentropic Euler-Maxwell equation extending the results of Duan [2]. In contrast to Duan, we suppose $A_{+} \neq A_{-}$, and we add the friction $\alpha_{ \pm}\left(u_{ \pm}-u_{+}\right)$where $\alpha_{+}=\left(\alpha / n_{+}\right)>0, \alpha_{-}=\left(\alpha / n_{-}\right)>0$, and $\alpha$ is a constant.

Theorem 1. Let $N \geq 4$ and (3) hold. There are $\delta_{0}>0$ and $C_{0}$ such that if

$$
\left\|\left[n_{ \pm 0}-1, u_{ \pm 0}, E_{0}, B_{0}\right]\right\|_{N} \leq \delta_{0},
$$

where $\|.\|_{N}$ is the $H^{N}$ norm, then the Cauchy problems (1) and (2) of the Euler-Maxwell system admit a unique global solution $\left[n_{ \pm}(t, x), u_{ \pm}(t, x), E(t, x), B(t, x)\right]$ with

$$
\begin{aligned}
& {\left[n_{ \pm}(t, x)-1, u_{ \pm}(t, x), E(t, x), B(t, x)\right] \in C\left([0, \infty) ; H^{N}\left(\mathbb{R}^{3}\right)\right) \cap \operatorname{Lip}\left([0, \infty) ; H^{N-1}\left(\mathbb{R}^{3}\right)\right),} \\
& \sup _{t \geq 0}\left\|\left[n_{ \pm}(t)-1, u_{ \pm}(t), E(t), B(t)\right]\right\|_{N} \leq C_{0}\left\|\left[n_{ \pm 0}-1, u_{ \pm 0}, E_{0}, B_{0}\right]\right\|_{N} .
\end{aligned}
$$

We obtain the decay rates of smooth solutions by the Fourier transform. The main results are stated as follows.

Theorem 2. There are $\delta_{1}>0$ and $C_{1}$ such that if

$$
\left\|\left[n_{ \pm 0}-1, u_{ \pm 0}, E_{0}, B_{0}\right]\right\|_{13}+\left\|\left[n_{ \pm 0}-1, u_{ \pm 0}, E_{0}, B_{0}\right]\right\|_{L^{1}} \leq \delta_{1},
$$

where $\|.\|_{13}$ is the $H^{13}$ norm, then the solution $\left[n_{ \pm}(t, x)\right.$, $\left.u_{ \pm}(t, x), E(t, x), B(t, x)\right]$ satisfies that for any $t \geq 0$,

$$
\begin{gathered}
\left|n_{ \pm}(t)\right|_{L^{q}} \leq C_{1}(1+t)^{-11 / 4}, \\
\left\|u_{ \pm}(t), E(t)\right\|_{L^{q}} \leq C_{1}(1+t)^{-2+(3 / 2 q)}, \\
\|B(t)\|_{L^{q}} \leq C_{1}(1+t)^{-(3 / 2)+(3 / 2 q),}
\end{gathered}
$$

with $2 \leq q \leq \infty$. Furthermore,

$$
\left\|U_{ \pm}(t)-G(t) * U_{ \pm 0}\right\|_{L^{q}} \leq C \varepsilon_{13}\left(V_{0}\right)(1+t)^{-2+(3 / 2 q)},
$$

where $G(t, x)$ is Green's matrix for the linearized system.

The proof of Theorem 1 and Theorem 2 is based on the energy method and the Fourier transform, as in [2]. There are three key steps: the first key step is the a priori estimate to establish the global solution and has the form

$$
\mathrm{E}_{N}(V(t))+\int_{0}^{t} \mathscr{D}_{N}(V(s)) \mathrm{d} s \leq \mathrm{E}_{N}\left(V_{0}\right),
$$

where $V(t)$ is the perturbation of solution (1) and $\mathrm{E}_{N}($.$) and \mathscr{D}_{N}($.$) denote the energy functional and energy$ dissipation rate functional as in [2]. This differs from $[4,10]$ because the two-fluid system has a more complex structure than one fluid, so obtaining energy estimates for the density, velocity, and electric magnetic fields for Euler-Maxwell require a different strategy. The time decay property of solutions to the nonlinear system requires the construction of functionals, capturing the optimal energy dissipation rate. The second key step is linearizing the homogeneous form of (1) and using the Fourier transform to obtain the $L^{p}-L^{q}$ time decay rate and the explicit representation of the solution. The third step is combining the previous two steps and applying the Fourier transform to obtain the time decay rate of the solution to the reformulated nonlinear system to finish the proof of Theorem 1. Thus, the solutions can be represented by the solution of the linearized system and the refined energy estimates using Duhamel's principle.

We introduce some notations that we will use later in this paper. For any integer $N \geq 0, H^{N}$ and $\dot{H}^{N}$ denote the Sobolev space $H^{N}\left(\mathbb{R}^{3}\right)$ and the $N^{\text {th }}$-order homogeneous Sobolev space, respectively. Set $L^{2}=H^{0}$. The norm of $H^{N}$ is denoted by $\|\cdot\|_{N}$ with $\|\cdot\|=\|\cdot\|_{0}$. The inner product in $L^{2}\left(\mathbb{R}^{3}\right)$ is denoted by $\langle\cdot, \cdot \cdot\rangle$, i.e., 


$$
\langle f, g\rangle=\int_{\mathbb{R}^{3}} f(x) g(x) \mathrm{d} x, \quad f=f(x), g=g(x) \in L^{2}\left(\mathbb{R}^{3}\right) .
$$

We denote $\partial^{\alpha}=\partial_{x_{1}}^{\alpha_{1}} \partial_{x_{2}}^{\alpha_{2}} \partial_{x_{3}}^{\alpha_{3}}=\partial_{1}^{\alpha_{1}} \partial_{2}^{\alpha_{2}} \partial_{3}^{\alpha_{3}}$ for the multi-in$\operatorname{dex} \alpha=\left[\alpha_{1}, \alpha_{2}, \alpha_{3}\right]$, and the length of $\alpha$ is $|\alpha|=\alpha_{1}+\alpha_{2}+\alpha_{3}$. In addition, $C$ and $\lambda$ denote some positive constants, where both $C$ and $\lambda$ may take different values in different places.

We organize this paper as follows. In Section 2, we reformulate the Cauchy problem and consider the proof of global existence and uniqueness of solutions. In Section 3, we discuss the time rate of decay for linearized systems, and we obtain the linearized system for $9 \times 9$. Finally, in Section 4 , we discuss the time decay rate of solutions of the nonlinear system (15) and complete the proof of Theorems and 2.

\section{Global Solution for the Nonlinear System}

2.1. Reformulation of the Problem. Denote by $\left[n_{ \pm}, u_{ \pm}, E, B\right]$ a smooth solution to system (1) with initial data (2) satisfying (3). Let

$$
\left\{\begin{array}{l}
\sigma_{ \pm}(t, x)=\sqrt{A_{ \pm}} \frac{2}{\gamma-1}\left(\left[n_{ \pm}\left(\frac{t}{\sqrt{\gamma}}, x\right)\right]^{(\gamma-1) / 2}-1\right), \\
v_{ \pm}=\frac{1}{\sqrt{\gamma}} u_{ \pm}\left(\frac{t}{\sqrt{\gamma}}, x\right) \\
\widetilde{E}=\frac{1}{\sqrt{\gamma}} E\left(\frac{t}{\sqrt{\gamma}}, x\right) \\
\widetilde{B}=\frac{1}{\sqrt{\gamma}} B\left(\frac{t}{\sqrt{\gamma}}, x\right) .
\end{array}\right.
$$

Define $V:=\left[\sigma_{ \pm}, v_{ \pm}, \widetilde{E}, \widetilde{B}\right]$ and

$$
\phi\left(\sigma_{ \pm}\right):=\left(\frac{\gamma-1}{2 \sqrt{A_{ \pm}}} \sigma_{ \pm}+1\right)^{2 /(\gamma-1)}-\frac{\sigma_{ \pm}}{\sqrt{A_{ \pm}}}-1 .
$$

Note that $V$ satisfies

$$
\left\{\begin{array}{l}
\partial_{t} \sigma_{ \pm}+\sqrt{A_{ \pm}}\left(\frac{\gamma-1}{2 \sqrt{A_{ \pm}}} \sigma_{ \pm}+1\right) \nabla \cdot v_{ \pm}+v_{ \pm} \cdot \nabla \sigma_{ \pm}=0, \\
\partial_{t} v_{ \pm}+v_{ \pm} \cdot \nabla v_{ \pm}+\sqrt{A_{ \pm}}\left(\frac{\gamma-1}{2 \sqrt{A_{ \pm}}} \sigma_{ \pm}+1\right) \nabla \sigma_{ \pm}= \pm\left(\frac{1}{\sqrt{\gamma}} \widetilde{E}+v_{ \pm} \times \widetilde{B}\right)-\frac{1}{\sqrt{\gamma}} v_{ \pm} v_{ \pm}-\frac{1}{\sqrt{\gamma}} \alpha_{ \pm}\left(v_{ \pm}-v_{\mp}\right), \\
\partial_{t} \widetilde{E}-\frac{1}{\sqrt{\gamma}} \nabla \times \widetilde{B}=\frac{1}{\sqrt{\gamma}} v_{-}+\frac{1}{\sqrt{\gamma}}\left(\phi\left(\sigma_{-}\right)+\frac{\sigma_{-}}{\sqrt{A_{-}}}\right) v_{-}-\frac{1}{\sqrt{\gamma}} v_{+}-\frac{1}{\sqrt{\gamma}}\left[\phi\left(\sigma_{+}\right)+\frac{\sigma_{+}}{\sqrt{A_{+}}}\right] v_{+}, \\
\partial_{t} \widetilde{B}-\frac{1}{\sqrt{\gamma}} \nabla \times \widetilde{E}=0, \\
\nabla \cdot \widetilde{E}=\frac{-1}{\sqrt{\gamma}}\left[\phi\left(\sigma_{-}\right)+\frac{\sigma_{-}}{\sqrt{A_{-}}}\right]+\frac{1}{\sqrt{\gamma}}\left[\phi\left(\sigma_{+}\right)+\frac{\sigma_{+}}{\sqrt{A_{+}}}\right] \\
\nabla . \widetilde{B}=0 \\
t>0, \\
x \in \mathbb{R}^{3}
\end{array}\right.
$$

with the initial data

$$
\left.V\right|_{t=0}=V_{0}:=\left[\sigma_{ \pm 0}, v_{ \pm 0}, \widetilde{E_{0}}, \widetilde{B_{0}}\right], \quad x \in \mathbb{R}^{3} .
$$

Here, we have used the notation $V_{0}=\left[\sigma_{ \pm 0}, v_{ \pm 0}, \widetilde{E_{0}}, \widetilde{B_{0}}\right]$ for the special case where $\left[n_{ \pm 0}, u_{ \pm 0}, E_{0}, B_{0}\right]$ is substituted into (13). Note that $V_{0}$ satisfies $\left\{\begin{array}{l}\nabla . \widetilde{E_{0}}=\frac{-1}{\sqrt{\gamma}}\left[\phi\left(\sigma_{-0}\right)+\frac{\sigma_{-0}}{\sqrt{A_{-}}}\right]+\frac{1}{\sqrt{\gamma}}\left[\phi\left(\sigma_{+0}\right)+\frac{\sigma_{+0}}{\sqrt{A_{+}}}\right], \\ \nabla . \widetilde{B_{0}}=0 \\ x \in \mathbb{R}^{3} .\end{array}\right.$ 
Suppose $U:=\left[n_{ \pm}, u_{ \pm}, E, B\right]$ is a smooth solution to the initial value problem of the original Cauchy problems (1) and (2), which satisfy (3). Now, we introduce another transformation by setting $\rho_{ \pm}(t, x)=n_{ \pm}(t, x)-1$, then $U:=\left[\rho_{ \pm}, u_{ \pm}, E, B\right]$ satisfies

$$
\left\{\begin{array}{l}
\partial_{t} \rho_{ \pm}+\nabla \cdot u_{ \pm}=-\nabla \cdot\left(\rho_{ \pm} u_{ \pm}\right) \\
\quad \partial_{t} u_{ \pm}+u_{ \pm} \mp E+A_{ \pm} \gamma \nabla \rho_{ \pm}=-u_{ \pm} \cdot \nabla u_{ \pm} \\
\quad-A_{ \pm} \gamma\left[\left(\rho_{ \pm}+1\right)^{\gamma-2}-1\right] \nabla \rho_{ \pm} \\
\pm\left(u_{ \pm} \times B\right)-\alpha_{ \pm}\left(u_{ \pm}-u_{\mp}\right) \\
\partial_{t} E-\nabla \times B-u_{-}+u_{+}=\rho_{-} u_{-}-\rho_{+} u_{+} \\
\partial_{t} B+\nabla \times E=0 \\
\nabla \cdot E=\rho_{+}-\rho_{-} \\
\nabla . B=0 \\
t>0 \\
x \in \mathbb{R}^{3}
\end{array}\right.
$$

with the initial data

$$
\left.U\right|_{t}=U_{0}:=\left[\rho_{ \pm 0}, u_{ \pm 0}, E_{0}, B_{0}\right], \quad x \in \mathbb{R}^{3},
$$

satisfying the compatibility condition

$$
\nabla \cdot E_{0}=\rho_{+0}-\rho_{-0}, \quad \nabla \cdot B_{0}=0,
$$

where $\rho_{ \pm 0}=n_{ \pm 0}-1$.

We will assume $N \geq 4$ is an integer. In addition to $V=\left[\sigma_{ \pm}, v_{ \pm}, \widetilde{E}, \widetilde{B}\right]$, define the full instant energy functional $\mathrm{E}_{N}(V(t))$ and the high-order instant energy functional $\mathrm{E}_{N}^{h}(V(t))$ via

$$
\begin{aligned}
& \left\{\begin{array}{l}
\varepsilon_{N}(V(t))=\|V(t)\|_{N}^{2}+k_{1} \sum_{|\alpha| \leq N-1}\left(\left\langle\partial^{\alpha} v_{+}, \nabla \partial^{\alpha} \sigma_{+}\right\rangle+\left\langle\partial^{\alpha} v_{-}, \nabla \partial^{\alpha} \sigma_{-}\right\rangle\right) \\
+k_{2} \sum_{|\alpha| \leq N-1}\left\langle\partial^{\alpha}\left(v_{+}-v_{-}\right), \nabla \partial^{\alpha} \widetilde{E}\right\rangle-k_{3} \sum_{|\alpha| \leq N-2}\left\langle\nabla \times \partial^{\alpha} \widetilde{E}, \partial^{\alpha} \widetilde{B}\right\rangle,
\end{array}\right. \\
& \left\{\begin{array}{l}
\varepsilon_{N}^{h}(V(t))=\|\nabla V(t)\|_{N-1}^{2}+k_{1} \sum_{1 \leq|\alpha| \leq N-1}\left(\left\langle\partial^{\alpha} v_{+}, \nabla \partial^{\alpha} \sigma_{+}\right\rangle+\left\langle\partial^{\alpha} v_{-}, \nabla \partial^{\alpha} \sigma_{-}\right\rangle\right) \\
+k_{2} \sum_{1 \leq|\alpha| \leq N-1}\left\langle\partial^{\alpha}\left(v_{+}-v_{-}\right), \nabla \partial^{\alpha} \widetilde{E}\right\rangle-k_{3} \sum_{1 \leq|\alpha| \leq N-2}\left\langle\nabla \times \partial^{\alpha} \widetilde{E}, \partial^{\alpha} \widetilde{B}\right\rangle,
\end{array}\right.
\end{aligned}
$$

where $0<k_{3} \ll k_{2} \ll k_{1} \ll 1$ are constants to be chosen later in the proof such that $k_{i},(i=1,2,3)$ are small enough compared to 1 and satisfy

$$
\begin{aligned}
& \mathscr{E}_{N}(V(t)) \sim\left\|\left[\sigma_{ \pm}, v_{ \pm}, \widetilde{E}, \widetilde{B}\right]\right\|_{N}^{2}, \\
& \mathscr{E}_{N}^{h}(V(t)) \sim\left\|\nabla\left[\sigma_{ \pm}, v_{ \pm}, \widetilde{E}, \widetilde{B}\right]\right\|_{N-1}^{2} .
\end{aligned}
$$
by

Define the dissipation rates $\mathscr{D}_{N}(V(t))$ and $\mathscr{D}_{N}^{h}(V(t))$ y

$$
\begin{aligned}
& \left\{\mathscr{D}_{N}(V(t))=\left\|\left[v_{+}, v_{-}\right]\right\|_{N}^{2}+\left\|\nabla\left[\sigma_{+}, \sigma_{-}\right]\right\|_{N-1}^{2}+\|\nabla[\widetilde{E}, \widetilde{B}]\|_{N-2}^{2}+\|\widetilde{E}\|^{2}+\left\|\sigma_{+}-\sigma_{-}\right\|^{2},\right. \\
& \left\{\mathscr{D}_{N}^{h}(V(t))=\left\|\nabla\left[v_{+}, v_{-}\right]\right\|_{N-1}^{2}+\left\|\nabla^{2}\left[\sigma_{+}, \sigma_{-}\right]\right\|_{N-2}^{2}+\left\|\nabla^{2}[\widetilde{E}, \widetilde{B}]\right\|_{N-3}^{2}+\|\nabla \widetilde{E}\|^{2}+\left\|\nabla\left(\sigma_{+}-\sigma_{-}\right)\right\|^{2} .\right.
\end{aligned}
$$

Proposition 1. Suppose initial data $V_{0}=\left[\sigma_{ \pm 0}, v_{ \pm 0}, \widetilde{E_{0}}, \widetilde{B_{0}}\right]$ $V \in C\left([0, \infty) ; H^{N}\left(\mathbb{R}^{3}\right)\right) \cap \operatorname{Lip}\left([0, \infty) ; H^{N-1}\left(\mathbb{R}^{3}\right)\right)$, satisfies (17). Then, there exist $\mathscr{C}_{N}(\cdot)$ and $\mathscr{D}_{N}(\cdot)$ having the forms (21) and (24), respectively, such that if $\mathscr{E}_{N}\left(V_{0}\right)>0$ is sufficiently small, the Cauchy problems (15) and (16) admit a unique, global, nonzero solution $V=\left[\sigma_{ \pm}, v_{ \pm}, \widetilde{E}, \widetilde{B}\right]$, satisfying

$$
\mathscr{E}_{N}(V(t))+\lambda \int_{0}^{t} \mathscr{D}_{N}(V(s)) \mathrm{d} s \leq \mathscr{C}_{N}\left(V_{0}\right),
$$

for any $t \geq 0$. 
Remark 1. The solutions obtained in Proposition 1 indeed represent the decay rates in time under some regularity and integrability conditions on initial data $V_{0}=\left[\sigma_{ \pm 0}, V_{ \pm 0}, \widetilde{E_{0}}, \widetilde{B_{0}}\right]$ and set

$$
\epsilon_{m}\left(V_{0}\right)=\left\|V_{0}\right\|_{m}+\left\|\left[v_{ \pm 0}, \widetilde{E_{0}}, \widetilde{B_{0}}\right]\right\|_{L^{1}},
$$

for the integer $m \geq 4$.

Remark 2. Note that the existence result in Theorem 1 follows from Proposition 1, the derivation of rates of (7) and (9) in Theorem 1, and Proposition 2. The proof of Proposition 2 is analogous to that of Lemma 5.2 in [10].
2.2. A Priori Estimates. In this section, we obtain uniformin-time a priori estimates for smooth solutions to the Cauchy problems (15) and (16) by using the classical energy method.

Theorem 3. Let $0<T \leq \infty$ be given. Suppose that $V=\left[\sigma_{ \pm}, v_{ \pm}, \widetilde{E}, \widetilde{B}\right] \in C\left([0, T) ; H^{N}\left(\mathbb{R}^{3}\right)\right)$ is smooth, that $\sigma$ satisfies

$$
\sup _{0 \leq t \leq T}\left\|\sigma_{ \pm}(t)\right\|_{N} \leq 1
$$

and that $V$ solves system (15) for $t \in(0, T)$. Then, there exist $\mathscr{E}_{N}($.$) and \mathscr{D}_{N}($.$) having the forms (21) and (24) such that for$ all $0 \leq t \leq T$,

$$
\frac{\mathrm{d}}{\mathrm{d} t} \mathscr{E}_{N}(V(t))+\lambda \mathscr{D}_{N}(V(t)) \leq C\left[\mathscr{E}_{N}(V(t))^{1 / 2}+\mathscr{E}_{N}(V(t))\right] \mathscr{D}_{N}(V(t))
$$

Proof. Performing the energy estimate, we obtain the following results:

Step 1 . We apply $\partial^{\alpha}$ to the first equation of (15) and then multiply that equation by $\partial^{\alpha} \sigma_{ \pm}$; also, we apply $\partial^{\alpha}$ to the second equation of (15) and then multiply that equation by $\partial^{\alpha} v_{ \pm}$; after many steps, we get

$$
\frac{1}{2} \frac{d}{d t}\|V\|_{N}^{2}+\frac{1}{\sqrt{\gamma}} v_{ \pm}\left\|\left[v_{+}, v_{-}\right]\right\|_{N}^{2} \leq C\|V\|_{N}\left(\left\|\left[v_{+}, v_{-}\right]\right\|^{2}+\left\|\nabla\left[\sigma_{+}, \sigma_{-}, v_{+}, v_{-}\right]\right\|_{N-1}^{2}\right)
$$

Step 2. We rewrite the first and second equations of (15) by putting the linear terms on the left-hand sides and the nonlinear terms on the right-hand sides:

$$
\begin{gathered}
\partial_{t} \sigma_{+}+\sqrt{A_{+}} \nabla \cdot v_{+}=-v_{+} \cdot \nabla \sigma_{+}-\frac{\gamma-1}{2} \sigma_{+} \nabla \cdot v_{+}:=f_{1}^{+}, \\
\partial_{t} \sigma_{-}+\sqrt{A_{-}} \nabla \cdot v_{-}=-v_{-} \cdot \nabla \sigma_{-}-\frac{\gamma-1}{2} \sigma_{-} \nabla \cdot v_{-}:=f_{1}^{-}, \\
\partial_{t} v_{+}+\sqrt{A_{+}} \nabla \sigma_{+}-\frac{1}{\sqrt{\gamma}} \widetilde{E}+\frac{v_{+}}{\sqrt{\gamma}} v_{+}+\frac{\alpha_{+}}{\sqrt{\gamma}}\left(v_{+}-v_{-}\right) \\
=-v_{+} \cdot \nabla v_{+}-\frac{\gamma-1}{2} \sigma_{+} \nabla \sigma_{+}+v_{+} \times \widetilde{B}:=f_{2}^{+}, \\
\partial_{t} v_{-}+\sqrt{A_{-}} \nabla \sigma_{-}+\frac{1}{\sqrt{\gamma}} \widetilde{E}+\frac{v_{-}}{\sqrt{\gamma}} v_{-}+\frac{\alpha_{-}}{\sqrt{\gamma}}\left(v_{-}-v_{+}\right) \\
=-v_{-} \cdot \nabla v_{-}-\frac{\gamma-1}{2} \sigma_{-} \nabla \sigma_{-}-v_{-} \times \widetilde{B}:=f_{2}^{-} .
\end{gathered}
$$

Let $|\alpha| \leq N-1$. If we apply $\partial^{\alpha}$ to (34), multiply by $\nabla \partial^{\alpha} \sigma_{+}$, integrate in $x$, and then combine the result with an application of $\partial^{\alpha}$ to (35) after which we multiply by $\nabla \partial^{\alpha} \sigma_{-}$and integrate in $x$, then we get 


$$
\begin{array}{r}
\frac{\mathrm{d}}{\mathrm{d} t} \mathscr{E}_{N, 1}^{\mathrm{int}}(V)+\lambda\left(\left\|\nabla\left[\sigma_{+}, \sigma_{-}\right]\right\|_{N-1}^{2}+\left\|\frac{\sigma_{+}}{\sqrt{A_{+}}}-\frac{\sigma_{-}}{\sqrt{A_{-}}}\right\|_{N}^{2}\right) \\
\leq C\left\|\left[v_{+}, v_{-}\right]\right\|_{N}^{2}+C\left\|\left[\sigma_{+}, \sigma_{-}, v_{+}, v_{-}, \widetilde{B}\right]\right\|_{N}^{2}\left\|\nabla\left[\sigma_{+}, \sigma_{-}, v_{+}, v_{-}\right]\right\|_{N-1}^{2},
\end{array}
$$

where

$\mathscr{E}_{N, 1}^{\text {int }}(V)=\sum_{|\alpha| \leq N-1}\left(\left\langle\partial^{\alpha} v_{+}, \nabla \partial^{\alpha} \sigma_{+}\right\rangle+\left\langle\partial^{\alpha} v_{-}, \nabla \partial^{\alpha} \sigma_{-}\right\rangle\right)$.

Step 3. We subtract equation (35) from equation (33) to get

$$
\begin{aligned}
\partial_{t}\left(v_{+}-v_{-}\right)+\left(\sqrt{A_{+}} \nabla \sigma_{+}-\sqrt{A_{-}} \nabla \sigma_{-}\right)+\frac{2}{\sqrt{\gamma}} \widetilde{E}= & -\partial^{\alpha}\left(v_{+} \cdot v_{+}\right)+\partial^{\alpha}\left(v_{+} \cdot v_{+}\right) \\
& -\frac{\gamma-1}{2} \partial^{\alpha}\left(\sigma_{+} \nabla \sigma_{+}\right)+\frac{\gamma-1}{2} \partial^{\alpha}\left(\sigma_{-} \nabla \sigma_{-}\right)-\partial^{\alpha}\left(v_{+} \times \widetilde{B}\right)-\partial^{\alpha}\left(v_{-} \times \widetilde{B}\right) \\
& -\frac{1}{\sqrt{\gamma}} \partial^{\alpha}\left(v_{+} v_{+}-v_{-} v_{-}\right)-\frac{1}{\gamma} \partial^{\alpha}\left[\alpha_{+}\left(v_{+}-v_{-}\right)-\alpha_{-}\left(v_{-}-v_{+}\right)\right] .
\end{aligned}
$$

Now, we apply $\partial^{\alpha}$ to (38), multiply by $\partial^{\alpha} E$, integrate by parts in $x$, and replace $\partial^{\alpha} \widetilde{E}$ with the third equation of (16). Then, we have

$$
\begin{aligned}
\frac{\mathrm{d}}{\mathrm{d} t} \mathscr{E}_{N, 2}^{\text {int }}(V)+\lambda\|\widetilde{E}\|_{N-1}^{2} \leq & C\left\|\left[v_{+}, v_{-}\right]\right\|_{N}^{2}+C\left\|\left[\sigma_{+}, \sigma_{-}\right]\right\|_{N-1}^{2}+C\left\|\left[v_{+}, v_{-}\right]\right\|_{N}\|\nabla \widetilde{B}\|_{N-2} \\
& +C\left\|\left[\sigma_{+}, \sigma_{-}, v_{+}, v_{-}, \widetilde{B}\right]\right\|_{N}^{2}\left\|\nabla\left[\sigma_{+}, \sigma_{-}, v_{+}, v_{-}\right]\right\|_{N-1}^{2},
\end{aligned}
$$

where

$\mathscr{E}_{N, 2}^{\text {int }}(v)=\sum_{|| \alpha \mid \leq N-1}\left\langle\partial^{\alpha}\left(v_{+}-v_{-}\right), \partial^{\alpha} \widetilde{E}\right\rangle \quad$ for $|\alpha| \leq N-1$.

Step 4. We apply $\partial^{\alpha}$ to the third equation of (15), multiply by $\partial^{\alpha} \nabla \times \widetilde{B}$, integrate by parts in $x$, and use the relation

$\left\|\partial^{\alpha} \partial_{x i} \widetilde{B}\right\|=\left\|\partial_{x i} \Delta^{-1} \nabla \times\left(\nabla \times \partial^{\alpha} \widetilde{B}\right)\right\| \leq\left\|\nabla \times \partial^{\alpha} \widetilde{B}\right\|$,

for each $1 \leq i \leq 3$. Then, we obtain

$$
\begin{aligned}
\frac{\mathrm{d}}{\mathrm{d} t} \mathscr{E}_{N, 3}^{\mathrm{int}}(V)+\lambda\|\nabla \widetilde{B}\|_{N-2}^{2} \leq & C\left\|\left[v_{+}, v_{-}, \widetilde{E}\right]\right\|_{N-1}^{2} \\
& +C\left\|\left[\sigma_{+}, \sigma_{-}\right]\right\|_{N}^{2}\left\|\nabla\left[v_{+}, v_{-}\right]\right\|_{N-1}^{2},
\end{aligned}
$$

where

$\mathscr{E}_{N, 3}^{\text {int }}(v)=-\sum_{|\alpha| \leq N-2}\left\langle\nabla \times \partial^{\alpha} \widetilde{E}, \partial^{\alpha} \widetilde{B}\right\rangle \quad$ for all $|\alpha| \leq N-2$.

Step 5. Utilizing steps (1)-(4) above, we can now prove (30). Define

$$
\mathscr{E}_{N}(V(t))=\|\nabla V(t)\|_{N}^{2}+\sum_{i=1}^{3} \mathscr{E}_{N}^{\text {int }}(V(t)),
$$

and note that constants $0<k_{3} \ll k_{2} \ll k_{1} \ll 1$ are to be determined. We observe that if $0<k_{i} \ll 1$ for $i=1,2,3$, are sufficiently small, then $\mathscr{E}_{N}(V) \sim\|V\|_{N}^{2}$ holds. Furthermore, by letting $0 \ll k_{3} \ll k_{2} \ll k_{1} \ll 1$ be sufficiently small, taking $k_{2}^{3 / 2} \ll k_{3}$, and taking the sum of (31), $k_{1} \times(36), k_{2} \times(42)$, and $k_{1} \times(42)$, we find that there exists $\lambda>0$ and $C>0$ such that (30) is satisfied: 


$$
\begin{aligned}
& \frac{1}{2} \frac{\mathrm{d}}{\mathrm{d} t}\|V\|_{N}^{2}+\frac{v_{ \pm}}{\sqrt{\gamma}}\left\|\left[v_{+}, v_{-}\right]\right\|_{N}^{2}+k_{1} \frac{\mathrm{d}}{\mathrm{d} t} \sum_{|\alpha| \leq N}\left(\left\langle\partial^{\alpha} v_{+}, \nabla \partial^{\alpha} \sigma_{+}\right\rangle+\left\langle\partial^{\alpha} v_{-}, \nabla \partial^{\alpha} \sigma_{-}\right\rangle\right) \\
& \quad+k_{1} \lambda\left(\left\|\nabla\left[\sigma_{+}, \sigma_{-}\right]\right\|_{N-1}^{2}+\left\|\frac{\sigma_{+}}{\sqrt{A_{+}}}-\frac{\sigma_{-}}{\sqrt{A_{-}}}\right\|_{N}^{2}\right)+k_{2} \frac{\mathrm{d}}{\mathrm{d} t} \sum_{|\alpha| \leq N-1}\left\langle\partial^{\alpha}\left(v_{+}-v_{-}\right), \partial^{\alpha} \widetilde{E}\right\rangle \\
& \quad+k_{2} \lambda\|\widetilde{E}\|_{N-1}^{2}-k_{3} \frac{\mathrm{d}}{\mathrm{d} t} \sum_{|\alpha| \leq N-2}\left\langle\nabla \times \partial^{\alpha} \widetilde{E}, \partial^{\alpha} \widetilde{B}\right\rangle+k_{3} \lambda\|\nabla \widetilde{B}\|_{N-2}^{2} \\
& \leq C\|V\|_{N}\left(\left\|\left[v_{+}, v_{-}\left\|^{2}+\right\| \nabla\left[\sigma_{+}, \sigma_{-}, v_{+}, v_{-}\right] \|_{N-1}^{2}\right)+k_{1} C\right\|\left[v_{+}, v_{-}\right] \|_{N}^{2}\right. \\
&+k_{1} C\left\|\left[\sigma_{+}, \sigma_{-}, v_{+}, v_{-}, \widetilde{B}\right]\right\|_{N}^{2}\left\|\nabla\left[\sigma_{+}, \sigma_{-}, v_{+}, v_{-}\right]\right\|_{N-1}^{2} \\
&+k_{2} C\left\|\left[v_{+}, v_{-}\right]\right\|_{N}^{2}+k_{2} C\left\|\left[\sigma_{+}, \sigma_{-}\right]\right\|_{N-1}^{2}+k_{2} C\left\|\left[v_{+}, v_{-}\right]\right\|_{N}\|\nabla \widetilde{B}\|_{N-2} \\
&+k_{2} C\left\|\left[\sigma_{+}, \sigma_{-}, v_{+}, v_{-}, \widetilde{B}\right]\right\|_{N}^{2}\left\|\nabla\left[\sigma_{+}, \sigma_{-}, v_{+}, v_{-}\right]\right\|_{N-1}^{2} \\
&+k_{3} C\left\|\left[v_{+}, v_{-}, \widetilde{E}\right]\right\|_{N-1}^{2}+k_{3} C\left\|\left[\sigma_{+}, \sigma_{-}\right]\right\|_{N}^{2}\left\|\nabla\left[v_{+}, v_{-}\right]\right\|_{N-1}^{2} .
\end{aligned}
$$

If we now let

then

$$
\begin{aligned}
\|V\| & \leq \mathscr{E}^{1 / 2}, \\
\left\|\left[\sigma_{+}, \sigma_{-}, v_{+}, v_{-}, \widetilde{B}\right]\right\|_{N}^{2} & =\mathscr{E}_{N}, \\
\left\|\nabla\left[\sigma_{+}, \sigma_{-}, v_{+}, v_{-}\right]\right\|_{N-1}^{2} & =\mathscr{D}_{N},
\end{aligned}
$$

$$
\begin{aligned}
& \frac{\mathrm{d}}{\mathrm{d} t}\left[\|V\|_{N}^{2}+k_{1} \sum_{|\alpha| \leq N}\left(\left\langle\partial^{\alpha} v_{+}, \nabla \partial^{\alpha} \sigma_{+}\right\rangle+\left\langle\partial^{\alpha} v_{-}, \nabla \partial^{\alpha} \sigma_{-}\right\rangle\right)+k_{2} \sum_{|\alpha| \leq N-1}\left\langle\partial^{\alpha}\left(v_{+}-v_{-}\right), \partial^{\alpha} E\right\rangle-k_{3} \frac{\mathrm{d}}{\mathrm{d} t} \sum_{|\alpha| \leq N-2}\left\langle\nabla \times \partial^{\alpha} \widetilde{E}, \partial^{\alpha} \widetilde{B}\right\rangle\right] \\
& \quad+\frac{v_{ \pm}}{\sqrt{\gamma}}\left\|\left[v_{+}, v_{-}\right]\right\|_{N}^{2}+k_{2} \lambda\left\|\nabla\left[\sigma_{+}, \sigma_{-}\right]\right\|_{N-1}^{2}+k_{3} \lambda\|\nabla \widetilde{B}\|_{N-2}^{2}+k_{2} \lambda\|\nabla \widetilde{E}\|_{N-2}^{2} \\
& \quad+k_{1} \lambda\left\|\frac{\sigma_{+}}{\sqrt{A_{+}}}-\frac{\sigma_{-}}{\sqrt{A_{-}}}\right\|_{N}^{2} \leq C\left[\|V\|_{N}\left\|\nabla\left[\sigma_{+}, \sigma_{-}, v_{+}, v_{-}\right]\right\|_{N-1}^{2}+k_{1} C\left\|\left[\sigma_{+}, \sigma_{-}, v_{+}, v_{-}, \widetilde{B}\right]\right\|_{N}^{2}\left\|\nabla\left[\sigma_{+}, \sigma_{-}, v_{+}, v_{-}\right]\right\|_{N-1}^{2}\right] .
\end{aligned}
$$

\section{It follows that}

$$
\begin{aligned}
& \frac{\mathrm{d}}{\mathrm{d} t} \mathscr{E}_{N}(V(t))+\frac{\nu_{ \pm}}{\sqrt{\gamma}}\left\|\left[v_{+}, v_{-}\right]\right\|_{N}^{2}+k_{2} \lambda\left\|\nabla\left[\sigma_{+}, \sigma_{-}\right]\right\|_{N-1}^{2}+k_{3} \lambda\|\nabla \widetilde{B}\|_{N-2}^{2} \\
& \quad+k_{2} \lambda\|\nabla \widetilde{E}\|_{N-2}^{2}+k_{2} \lambda\|\widetilde{E}\|^{2}+k_{1} \lambda\left\|\frac{\sigma_{+}}{\sqrt{A_{+}}}-\frac{\sigma_{-}}{\sqrt{A_{-}}}\right\|_{N}^{2} \leq C\left[\left(\|V\|_{N}+\left\|\nabla\left[\sigma_{+}, \sigma_{-}, v_{+}, v_{-}\right]\right\|_{N-1}^{2}\right)\left\|\nabla\left[\sigma_{+}, \sigma_{-}, v_{+}, v_{-}\right]\right\|_{N-1}^{2}\right] .
\end{aligned}
$$


Thus,

$$
\frac{\mathrm{d}}{\mathrm{d} t} \mathscr{E}_{N}(V(t))+\lambda \mathscr{D}_{N}(V(t)) \leq C\left[\mathscr{E}_{N}(V(t))^{1 / 2}+\mathscr{E}_{N}(V(t))\right] \mathscr{D}_{N}(V(t))
$$

and this concludes the proof.

2.3. Proof of Global Existence. We consider the global existence of the smooth solution to the isentropic Euler-Maxwell system for a quasilinear symmetric hyperbolic system (15). Therefore, we combine those a priori estimates with the local existence of solutions to extend the local solution up to infinite time by using the continuity of $\mathscr{E}_{N}(V(t))$.

Lemma 1 (local existence of smooth solution, see $[2,13,14])$. Assume $V_{0} \in H^{N}\left(\mathbb{R}^{3}\right)$ satisfies (17). Then, there exists $T_{0}>0$ such that the Cauchy problems (15) and (16) admit a unique solution on $\left[0, T_{0}\right)$ with

$$
V \in C\left(\left[0, T_{0}\right) ; H^{N}\left(\mathbb{R}^{3}\right)\right) \cap \operatorname{Lip}\left(\left[0, T_{0}\right) ; H^{N-1}\left(\mathbb{R}^{3}\right)\right) .
$$

Proof of Proposition. 1. Since (15) is a quasilinear symmetric hyperbolic system, the global existence of smooth solutions follows from the local existence result in Lemma 1 (see also Section 16 of [14]). In addition, the a priori estimate (30) in Theorem 3 and the continuity argument show that $\mathscr{E}_{N}(V(t))$ is bounded uniformly in time under the assumption that $\mathscr{E}_{N}\left(V_{0}\right)>0$ is sufficiently small. Therefore, global solutions satisfying (26) and (27) exist. This concludes the proof of Proposition 1.

\section{Linearized Homogeneous System}

3.1. Linearized Equations. To obtain the time decay rates of a solution to the nonlinear system (15) or (18), we consider the linearized homogeneous equations of system (18):

$$
\left\{\begin{array}{l}
\partial_{t} \rho_{ \pm}+\nabla \cdot u_{ \pm}=0, \\
\partial_{t} u_{ \pm}+u_{ \pm}+\alpha_{ \pm} u_{ \pm}-\alpha_{ \pm} u_{\mp} \mp E+A_{ \pm} \gamma \nabla \rho_{ \pm}=0, \\
\partial_{t} E-\nabla \times B-u_{-}+u_{+}=0, \\
\partial_{t} B+\nabla \times E=0, \\
\nabla . E=\rho_{+}-\rho_{-} \\
\nabla . B=0 \\
t>0 \\
x \in \mathbb{R}^{3}
\end{array}\right.
$$

with the given initial data

$$
\left.U\right|_{t=0}=U_{0}:=\left[\rho_{ \pm 0}, u_{ \pm 0}, E_{0}, B_{0}\right], \quad x \in \mathbb{R}^{3},
$$

which satisfies the compatibility conditions

$$
\nabla \cdot E_{0}=\rho_{+0}-\rho_{-0}, \quad \nabla \cdot B_{0}=0, x \in \mathbb{R}^{3} .
$$

Throughout this section, we let $U=\left[\rho_{ \pm}, u_{ \pm}, E, B\right]$ be the solution to system (51). Moreover, in this section, we introduce some notation about Fourier transform $f: \mathbb{R}^{3} \longrightarrow \mathbb{R}$, defined by

$$
\begin{aligned}
& \hat{f}(k)=\int_{\mathbb{R}^{3}} e^{-i x \cdot k} f(x) \mathrm{d} x, \\
& x \cdot k:=\sum_{j=1}^{3} x_{j} k_{j}, \quad 4 k \in \mathbb{R}^{3},
\end{aligned}
$$

where $i$ is the complex number, and we use the energy method to the initial value problems (51) and (53) in Fourier space to show that there is a time-frequency Lyapunov inequality, which leads to the pointwise time-frequency upper-bound of the solution.

We will use the energy method to the initial value problems (51) and (53) in the Fourier transform to show that there is a time-frequency Lyapunov functional which is equivalent to $|\widehat{U(t, k)}|^{2}$ and moreover its dissipation rate can be represented by itself.

3.2. Representation of Solution. Denote by $U=\left[\rho_{ \pm}, u_{ \pm}, E, B\right]=e^{t L} U_{0}$ the explicit solution to the Cauchy problems (51) and (52), satisfying (53). In this section, we study the representation of $U$.

First, we take the time derivative for the first equation and the divergence of the second equation of system (51) and substitute $\nabla \cdot E=\rho_{+}-\rho_{-}$. So,

$$
\partial_{t t} \rho_{ \pm}+\partial_{t} \nabla \cdot u_{ \pm}=0
$$

$$
\nabla \cdot \partial_{t} u_{ \pm}+\nabla \cdot u_{ \pm}+\alpha_{ \pm} \nabla \cdot\left(u_{ \pm}-u_{\mp}\right) \mp \nabla \cdot E+A_{ \pm} \gamma \Delta \rho_{ \pm}=0 .
$$

By combining the two equations (55) and (56), we have

$$
\partial_{t t} \rho_{ \pm}+\left(1+\alpha_{ \pm}\right) \partial_{t} \rho_{ \pm}-\alpha_{ \pm} \partial_{t} \rho_{\mp} \pm\left(\rho_{+}-\rho_{-}\right)-A_{ \pm} \gamma \Delta \rho_{ \pm}=0 \text {, }
$$

with the initial data given by

$$
\left\{\begin{array}{l} 
\pm\left.\left(\rho_{+}-\rho_{-}\right)\right|_{t=0}= \pm\left(\rho_{+0}-\rho_{-0}\right)= \pm \nabla \cdot E_{0} \\
\left.\partial_{t} \rho_{ \pm}\right|_{t=0}=-\nabla \cdot u_{ \pm 0} .
\end{array}\right.
$$


Then, taking the Fourier transform of the second-order ODE (57) with (58), we get

$$
\left\{\begin{array}{l}
\partial_{t t} \widehat{\rho}_{ \pm}+\left(1+\alpha_{ \pm}\right) \partial_{t} \widehat{\rho}_{ \pm}-\alpha_{ \pm} \partial_{t} \widehat{\rho}_{\mp} \pm\left(\widehat{\rho}_{+}-\widehat{\rho}_{-}\right)+A_{ \pm} \gamma|k|^{2} \widehat{\rho}_{ \pm}=0, \\
\pm\left.\left(\widehat{\rho}_{+}-\widehat{\rho}_{-}\right)\right|_{t=0}= \pm\left(\widehat{\rho}_{+0}-\widehat{\rho}_{-0}\right)= \pm i k \cdot \widehat{E}_{0}, \\
\left.\partial_{t} \widehat{\rho}_{ \pm}\right|_{t=0}=-i k \cdot \widehat{u}_{ \pm 0} .
\end{array}\right.
$$

Now, set

$$
\begin{aligned}
x_{1} & =\hat{\rho}_{+}, \\
x_{2} & =\dot{x}_{1}=\partial_{t} \hat{\rho}_{+}, \\
x_{3} & =\hat{\rho}_{-}, \\
x_{4} & =\dot{x}_{3}=\partial_{t} \hat{\rho}_{-}, \\
x & =\left[x_{1}, x_{2}, x_{3}, x_{4}\right]^{T} .
\end{aligned}
$$

Then,

$$
x_{t}=A(k) x,
$$

where

$$
A(k)=\left[\begin{array}{cccc}
0 & 1 & 0 & 0 \\
-1-A_{+} \gamma|k|^{2} & -\left(1+\alpha_{+}\right) & 1 & \alpha_{+} \\
0 & 0 & 0 & 1 \\
1 & \alpha_{-} & -1-A_{-} \gamma|k|^{2} & -\left(1+\alpha_{-}\right)
\end{array}\right] .
$$

Note that the eigenvector of the matrix $A(k)$ is given by

$$
\begin{aligned}
& a_{1}(\lambda)=\left[\begin{array}{c}
\left(1+A_{-} \gamma|k|^{2}\right)+\left(1+\alpha_{-}\right) \lambda+\lambda^{2} \\
{\left[\left(1+A_{-} \gamma|k|^{2}\right)+\left(1+\alpha_{-}\right) \lambda+\lambda^{2}\right] \lambda} \\
1+\alpha_{-} \lambda \\
\left(1+\alpha_{-} \lambda\right) \lambda \\
1+\alpha_{+} \lambda \\
\left(1+\alpha_{+} \lambda\right) \lambda
\end{array}\right], \\
& a_{2}(\lambda)=\left[\begin{array}{c} 
\\
\left(1+A_{+} \gamma|k|^{2}\right)+\left(1+\alpha_{+}\right) \lambda+\lambda^{2} \\
{\left[\left(1+A_{+} \gamma|k|^{2}\right)+\left(1+\alpha_{+}\right) \lambda+\lambda^{2}\right] \lambda}
\end{array}\right] .
\end{aligned}
$$

In the next two sections, we provide an estimate for $\widehat{u}_{ \pm}, \widehat{\rho}_{ \pm}, \widehat{E}$, and $\widehat{B}$. In Section 3.2.1, we estimate for $\widehat{u}_{ \pm}$and $\widehat{\rho}_{ \pm}$, and in Section 3.2.2, we estimate for $\widehat{u}_{+}, \widehat{E}$, and $\widehat{B}$. To do so, we set $\widetilde{k}=k /|k|$, and we use the relation $\widehat{u}_{+}=\widetilde{k} \widetilde{k} \cdot \widehat{u}_{+}-\widetilde{k} \times$ $\left(\widetilde{k} \times \widehat{u}_{ \pm}\right)$where we refer to $\widetilde{k} \widetilde{k} \cdot \widehat{u}_{ \pm}$as the "parallel part" and $\widetilde{k} \times\left(\tilde{k} \times \widehat{u}_{ \pm}\right)$as the "perpendicular part."

3.2.1. Parallel Part. We proceed with the asymptotic expansion of eigenvalues: let $\lambda_{j}(k), j=1,2,3,4$, be the eigenvalues of the matrix $A(k)$. Taking the determinant, we see the eigenvalues satisfy

$$
\begin{aligned}
\operatorname{det}(A-\lambda I)= & \lambda^{4}+\left(2+\alpha_{+}+\alpha_{-}\right) \lambda^{3}+\left(3+\alpha_{+}+\alpha_{-}+A_{+} \gamma|k|^{2}\right. \\
& \left.+A_{-} \gamma|k|^{2}\right) \lambda^{2} \\
& +\left(2+A_{+} \gamma|k|^{2}+A_{-} \gamma|k|^{2}+\alpha_{-} A_{+} \gamma|k|^{2}+\alpha_{+} A_{-} \gamma|k|^{2}\right) \lambda \\
& +A_{+} \gamma|k|^{2}+A_{-} \gamma|k|^{2}+A_{-} A_{+} \gamma^{2}|k|^{4}=0 .
\end{aligned}
$$

(i) First we consider when $|k| \longrightarrow 0$, since $\operatorname{det}(A-\lambda I)=$ 0 satisfies

$$
\lambda(\lambda+1)\left(\lambda^{2}+\left(1+\alpha_{-}+\alpha_{+}\right) \lambda+2\right)=0,
$$

$\lambda_{j}(k)$ has the following asymptotic expansion:

$$
\lambda_{j}(k)=\lambda_{j}^{(0)}+\lambda_{j}^{(1)} k+\lambda_{j}^{(2)} k^{2}+\ldots,
$$

where each coefficient $\lambda_{j}^{(n)}$ is given by direct computation as

$\lambda_{1}^{(0)}=\lambda_{1}^{(1)}=0$,

$\lambda_{1}^{(2)}=-\frac{1}{2}\left(A_{+} \gamma+A_{-} \gamma\right)$,

$\lambda_{2}^{(0)}=-1$,

$\lambda_{2}^{(1)}=0$,

$\lambda_{2}^{(2)}=-\frac{A_{+} \gamma+A_{-} \gamma+\alpha_{-} A_{+} \gamma+\alpha_{+} A_{-} \gamma}{2-\alpha_{+}-\alpha_{-}}$,

$\lambda_{3}^{(0)}=\frac{-\left(1+\alpha_{+}+\alpha_{-}\right)-\sqrt{\left(1+\alpha_{+}+\alpha_{-}\right)^{2}-8}}{2}$

$\lambda_{3}^{(1)}=0$,

$\lambda_{3}^{(2)}=0$,

$\lambda_{4}^{(0)}=\frac{-\left(1+\alpha_{+}+\alpha_{-}\right)+\sqrt{\left(1+\alpha_{+}+\alpha_{-}\right)^{2}-8}}{2}$,

$\lambda_{4}^{(1)}=0$,

$\lambda_{4}^{(2)}=0$.

Thus, the approximation of the eigenvalue when $|k| \longrightarrow 0$ is 


$$
\begin{aligned}
& \lambda_{1}=-\frac{1}{2}\left(A_{+} \gamma+A_{-} \gamma\right) k^{2} \\
& \lambda_{2}=-1-\left[\frac{A_{+} \gamma+A_{-} \gamma+\alpha_{-} A_{+} \gamma+\alpha_{+} A_{-} \gamma}{2-\alpha_{+}-\alpha_{-}}\right] k^{2}, \\
& \lambda_{3}=\frac{-\left(1+\alpha_{+}+\alpha_{-}\right)-\sqrt{\left(1+\alpha_{+}+\alpha_{-}\right)^{2}-8}}{2} \\
& \lambda_{4}=\frac{-\left(1+\alpha_{+}+\alpha_{-}\right)+\sqrt{\left(1+\alpha_{+}+\alpha_{-}\right)^{2}-8}}{2}
\end{aligned}
$$

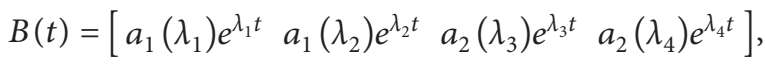

then the Green matrix $G$ for (61) is given by $G(t)=B(t) B^{-1}(0)$, and the solution is

$$
x=G(t) x_{0}
$$

Thus, after a series of calculation using symbolic manipulator, we have

Therefore, if we define

$$
\left[\begin{array}{l}
x_{1} \\
x_{2} \\
x_{3} \\
x_{4}
\end{array}\right]=\left[\begin{array}{cccc}
\left(O(1) e^{-\theta t}+O(1) e^{-\theta k^{2} t}\right) & \left(O(1) e^{-\theta t}+O(1) e^{-\theta k^{2} t}\right) & \left(O(1) e^{-\theta t}+O(1) e^{-\theta k^{2} t}\right) & \left(O(1) e^{-\theta t}+O(1) e^{-\theta k^{2} t}\right) \\
\left(O(1) e^{-\theta t}+O(1) e^{-\theta k^{2} t} k^{2}\right) & \left(O(1) e^{-\theta t}+O(1) e^{-\theta k^{2} t} k^{2}\right) & \left(O(1) e^{-\theta t}+O(1) e^{-\theta k^{2} t} k^{2}\right) & \left(O(1) e^{-\theta t}+O(1) e^{-\theta k^{2} t} k^{2}\right) \\
\left(O(1) e^{-\theta t}+O(1) e^{-\theta k^{2} t}\right) & \left(O(1) e^{-\theta t}+O(1) e^{-\theta k^{2} t}\right) & \left(O(1) e^{-\theta t}+O(1) e^{-\theta k^{2} t}\right) & \left(O(1) e^{-\theta t}+O(1) e^{-\theta k^{2} t}\right) \\
\left(O(1) e^{-\theta t}+O(1) e^{-\theta k^{2} t} k^{2}\right) & \left(O(1) e^{-\theta t}+O(1) e^{-\theta k^{2} t} k^{2}\right) & \left(O(1) e^{-\theta t}+O(1) e^{-\theta k^{2} t} k^{2}\right) & \left(O(1) e^{-\theta t}+O(1) e^{-\theta k^{2} t} k^{2}\right)
\end{array}\right]\left[\begin{array}{c}
x_{2,0} \\
x_{3,0} \\
x_{4,0}
\end{array}\right],
$$

where $\theta$ is the sum of real parts of $\lambda_{j}, j=1,2,3,4$. Thus,

$$
\begin{aligned}
\hat{\rho}_{+}(t, k)= & \left(O(1) e^{-\theta t}+O(1) e^{-\theta k^{2} t}\right) \widehat{\rho}_{+0}-\left(O(1) e^{-\theta t}+O(1) e^{-\theta k^{2} t}\right) i k \cdot \widehat{u}_{+0} \\
& +\left(O(1) e^{-\theta t}+O(1) e^{-\theta k^{2} t}\right) \widehat{\rho}_{-0}-\left(O(1) e^{-\theta t}+O(1) e^{-\theta k^{2} t}\right) i k \cdot \widehat{u}_{-0}, \\
-i k \cdot \widehat{u}_{+}(t, k)= & \left(O(1) e^{-\theta t}+O(1) e^{-\theta k^{2} t} k^{2}\right) \widehat{\rho}_{+0}-\left(O(1) e^{-\theta t}+O(1) e^{-\theta k^{2} t} k^{2}\right) i k \cdot \widehat{u}_{+0} \\
& +\left(O(1) e^{-\theta t}+O(1) e^{-\theta k^{2} t} k^{2}\right) \widehat{\rho}_{-0}-\left(O(1) e^{-\theta t}+O(1) e^{-\theta k^{2} t} k^{2}\right) i k \cdot \widehat{u}_{-0}, \\
& +\left(O(1) e^{-\theta k^{2} t}+O(1) e^{-\theta t}\right) \widehat{\rho}_{-0}-\left(O(1) e^{-\theta k^{2} t}+O(1) e^{-\theta t}\right) i k \cdot \widehat{u}_{-0}, \\
\widehat{\rho}_{-}(t, k)= & \left(O(1) e^{-\theta k^{2} t}+O(1) e^{-\theta t}\right) \widehat{\rho}_{+0}-\left(O(1) e^{-\theta k^{2} t}+O(1) e^{-\theta t}\right) i k \cdot \widehat{u}_{+0} \\
-i k \cdot \widehat{u}_{-}(t, k)= & \left(O(1) e^{-\theta t}+O(1) e^{-\theta k^{2} t} k^{2}\right) \widehat{\rho}_{+0}-\left(O(1) e^{-\theta t}+O(1) e^{-\theta k^{2} t} k^{2}\right) i k \cdot \widehat{u}_{+0} \\
& +\left(O(1) e^{-\theta t}+O(1) e^{-\theta k^{2} t} k^{2}\right) \widehat{\rho}_{-0}-\left(O(1) e^{-\theta t}+O(1) e^{-\theta k^{2} t} k^{2}\right) i k \cdot \widehat{u}_{-0} .
\end{aligned}
$$

In fact, we know that

$\widehat{u}_{ \pm}=\widetilde{k} \widetilde{k} \cdot \widehat{u}_{ \pm}-\widetilde{k} \times\left(\widetilde{k} \times \widehat{u}_{ \pm}\right)$implies $\widetilde{k} \widetilde{k} \cdot \widehat{u}_{ \pm}=\widehat{u}_{ \pm}+\widetilde{k} \times\left(\widetilde{k} \times \widehat{u}_{ \pm}\right)$.
Therefore, plugging (73) in the second and the fourth equations of (72), we obtain 


$$
\begin{aligned}
& \widehat{u}_{+}(t, k)=-\widetilde{k} \times\left(\widetilde{k} \times \widehat{u}_{+}\right)+\left(\begin{array}{c}
\frac{i \widetilde{k}}{|k|}\left(O(1) e^{-\theta t}+O(1) e^{-\theta k^{2} t} k^{2}\right) \widehat{\rho}_{+0}+\left(O(1) e^{-\theta t}+O(1) e^{-\theta k^{2} t} k^{2}\right) \tilde{k} \widetilde{k} \cdot \widehat{u}_{+0} \\
i \widetilde{k} \\
+\frac{|k|}{|k|}\left(O(1) e^{-\theta t}+O(1) e^{-\theta k^{2} t} k^{2}\right) \widehat{\rho}_{-0}+\left(O(1) e^{-\theta t}+O(1) e^{-\theta k^{2} t} k^{2}\right) \widetilde{k} \widetilde{k} \cdot \widehat{u}_{-0}
\end{array}\right), \\
& \widehat{u}_{-}(t, k)=-\widetilde{k} \times\left(\widetilde{k} \times \widehat{u}_{-}\right)+\left(\begin{array}{c}
\frac{i \widetilde{k}}{|k|}\left(O(1) e^{-\theta t}+O(1) e^{-\theta k^{2} t} k^{2}\right) \widehat{\rho}_{+0}+\left(O(1) e^{-\theta t}+O(1) e^{-\theta k^{2} t} k^{2}\right) \tilde{k} \widetilde{k} \cdot \widehat{u}_{+0} \\
+\frac{\tilde{k}}{|k|}\left(O(1) e^{-\theta t}+O(1) e^{-\theta k^{2} t} k^{2}\right) \widehat{\rho}_{-0}+\left(O(1) e^{-\theta t}+O(1) e^{-\theta k^{2} t} k^{2}\right) \widetilde{k} \widetilde{k} \cdot \widehat{u}_{-0}
\end{array}\right) \text {. }
\end{aligned}
$$

(ii) When $|k| \longrightarrow \infty, \lambda_{j}(k)$ has the following asymptotic expansion:

$$
\lambda_{j}(k)=\mu_{j}^{(1)} k+\mu_{j}^{(0)}+\mu_{j}^{(-1)} k^{-1}+\mu_{j}^{(-2)} k^{-2}+\mu_{j}^{(-3)} k^{-3}+\ldots,
$$

where each coefficient $\mu_{j}^{(n)}$ is given by direct computation as

$$
\begin{aligned}
& \mu_{1}^{(1)}=i \sqrt{A_{-} \gamma}, \\
& \mu_{1}^{(0)}=-\frac{\left(1+\alpha_{-}\right)}{2}, \\
& \mu_{2}^{(1)}=-i \sqrt{A_{-} \gamma}, \\
& \mu_{2}^{(0)}=-\frac{\left(1+\alpha_{-}\right)}{2}, \\
& \mu_{3}^{(1)}=i \sqrt{A_{+} \gamma}, \\
& \mu_{3}^{(0)}=-\frac{\left(1+\alpha_{+}\right)}{2}, \\
& \mu_{4}^{(1)}=-i \sqrt{A_{+} \gamma}, \\
& \mu_{4}^{(0)}=-\frac{\left(1+\alpha_{+}\right)}{2} .
\end{aligned}
$$

So, $G=B(t) B^{-1}(0)$ is the Green matrix for (61), and the solution is

$$
x=G(t) x_{0} .
$$

Thus, after a series of calculation using the symbolic manipulator, we obtain

$$
\begin{aligned}
& {\left[\begin{array}{l}
x_{1} \\
x_{2} \\
x_{3} \\
x_{4}
\end{array}\right]=\left[\begin{array}{cccc}
O(1) e^{-\theta t} & O(1) e^{-\theta t}|k|^{-1} & O(1) e^{-\theta t}|k|^{-1} & O(1) e^{-\theta t} \\
O(1) e^{-\theta t}|k| & O(1) e^{-\theta t} & O(1) e^{-\theta t} & O(1) e^{-\theta t}|k|^{-1} \\
O(1) e^{-\theta t}|k|^{-1} & O(1) e^{-\theta t} & O(1) e^{-\theta t} & O(1) e^{-\theta t}|k|^{-1} \\
O(1) e^{-\theta t} & O(1) e^{-\theta t}|k|^{-1} & O(1) e^{-\theta t}|k| & O(1) e^{-\theta t}
\end{array}\right]\left[\begin{array}{c}
x_{1,0} \\
x_{2,0} \\
x_{3,0} \\
x_{4,0}
\end{array}\right] \text {, }} \\
& \widehat{\rho}_{+}(t, k)=O(1) e^{-\theta t} \widehat{\rho}_{+0}-O(1) e^{-\theta t}|k|^{-1} i k \cdot \widehat{u}_{+0}+O(1) e^{-\theta t}|k|^{-1} \widehat{\rho}_{-0}-O(1) e^{-\theta t} i k \cdot \widehat{u}_{-0}, \\
& -i k \cdot \widehat{u}_{+}(t, k)=O(1) e^{-\theta t}|k| \widehat{\rho}_{+0}-O(1) e^{-\theta t} i k \cdot \widehat{u}_{+0}+O(1) e^{-\theta t} \widehat{\rho}_{-0}-O(1) e^{-\theta t}|k|^{-1} i k \cdot \widehat{u}_{-0} \text {, } \\
& \hat{\rho}_{-}(t, k)=O(1) e^{-\theta t}|k|^{-1} \hat{\rho}_{+0}-O(1) e^{-\theta t} i k \cdot \widehat{u}_{+0}+O(1) e^{-\theta t} \widehat{\rho}_{-0}-O(1) e^{-\theta t}|k|^{-1} i k \cdot \widehat{u}_{-0}, \\
& -i k \cdot \widehat{u}_{-}(t, k)=O(1) e^{-\theta t} \widehat{\rho}_{+0}-O(1) e^{-\theta t}|k|^{-1} i k \cdot \widehat{u}_{+0}+O(1) e^{-\theta t}|k| \widehat{\rho}_{-0}-O(1) e^{-\theta t} i k \cdot \widehat{u}_{-0} \text {. }
\end{aligned}
$$


Therefore, after plugging (72) into the second and fourth equations of (79), we obtain

$$
\begin{aligned}
& \widehat{u}_{+}(t, k)=-\tilde{k} \times\left(\widetilde{k} \times \widehat{u}_{+}\right)+\left(O(1) e^{-\theta t} \tilde{i} \tilde{k} \tilde{k} \cdot \widehat{\rho}_{+0}+O(1) e^{-\theta t} \tilde{k} \widetilde{k} \cdot \widehat{u}_{+0}+O(1) e^{-\theta t} \frac{i \tilde{k}}{|k|} \widehat{\rho}_{-0}+O(1) e^{-\theta t} \frac{\widetilde{k} \tilde{k}}{|k|} \cdot \widehat{u}_{-0}\right), \\
& \widehat{u}_{-}(t, k)=-\tilde{k} \times\left(\widetilde{k} \times \widehat{u}_{-}\right)+\left(O(1) e^{-\theta t} \frac{i \widetilde{k}}{|k|} \widehat{\rho}_{+0}+O(1) e^{-\theta t} \frac{\widetilde{k} \widetilde{k}}{|k|} \cdot \widehat{u}_{+0}+O(1) e^{-\theta t} i \widetilde{k} \widetilde{k} \widehat{\rho}_{-0}+O(1) e^{-\theta t} \tilde{k} \widetilde{k} \cdot \widehat{u}_{-0}\right) .
\end{aligned}
$$

(iii) When $0<|k|<\infty$, we consider the Routh-Hurwitz stability condition of the characteristic polynomial (64). That is, if we write (64) in the form

$$
b_{0} \lambda^{4}+b_{1} \lambda^{3}+b_{2} \lambda^{2}+b_{3} \lambda+b_{4}=0
$$

The system stability requires

$$
\begin{aligned}
b_{1} & >0 \\
b_{1} b_{2}-b_{0} b_{3} & >0 \\
\left.\left(b_{1} b_{2}-b_{0} b_{3}\right)\right) b_{3}-b_{1}^{2} b_{4} & >0 \\
b_{4} & >0
\end{aligned}
$$

$$
\begin{aligned}
& \left(2+\alpha_{+}+\alpha_{-}\right)>0, \\
& {\left[\left(2+\alpha_{+}+\alpha_{-}\right)\left(3+\alpha_{+}+\alpha_{-}+A_{+} \gamma|k|^{2}+A_{-} \gamma|k|^{2}\right)\right]-\left(2+A_{+} \gamma|k|^{2}+A_{-} \gamma|k|^{2}+\alpha_{-} A_{+} \gamma|k|^{2}+\alpha_{+} A_{-} \gamma|k|^{2}\right)>0,} \\
& \quad\left[\left(4+2 \alpha_{+}+2 \alpha_{-}+A_{+} \gamma|k|^{2}+A_{-} \gamma|k|^{2}+3 \alpha_{+}+\alpha_{+}^{2}+2 \alpha_{+} \alpha_{-}+\alpha_{+} A_{+} \gamma|k|^{2}+3 \alpha_{-}+\alpha_{-}^{2}+\alpha_{-} A_{-} \gamma|k|^{2}\right)\right. \\
& \left.\quad \cdot\left(2+A_{+} \gamma|k|^{2}+A_{-} \gamma|k|^{2}+\alpha_{-} A_{+} \gamma|k|^{2}+\alpha_{+} A_{-} \gamma|k|^{2}\right)\right] \\
& -\left[\left(2+\alpha_{+}+\alpha_{-}\right)^{2}\left(A_{+} \gamma|k|^{2}+A_{-} \gamma|k|^{2}+A_{-} A_{+} \gamma^{2}|k|^{4}\right)\right]>0, \\
& A_{+} \gamma|k|^{2}+A_{-} \gamma|k|^{2}+A_{-} A_{+} \gamma^{2}|k|^{4}>0 .
\end{aligned}
$$

It is not difficult to show that the above inequalities are satisfied, and this implies that all roots of the characteristic equation have negative real parts.

Although the eigenvalues may coalesce, the computations in (i), (ii), and (iii) show that coalescence occurs when the real parts of the eigenvalues are negative. Therefore, the stability conditions are satisfied.

\subsubsection{Perpendicular Part. Now, we consider}

It can be shown that, in our case, the conditions corresponding to (82) are given, respectively, by 
Now, taking the Fourier Transform and multiplying by $-i k$, we obtain

$$
\left\{\begin{array}{l}
\partial_{t} M_{1}+M_{1}+\alpha_{+} M_{1}-\alpha_{+} M_{2}-M_{3}=0, \\
\partial_{t} M_{2}+M_{2}+\alpha_{-} M_{2}-\alpha_{-} M_{1}+M_{3}=0, \\
\partial_{t} M_{3}-i k \times M_{4}+M_{1}-M_{2}=0, \\
\partial_{t} M_{4}+i k \times M_{3}=0 .
\end{array}\right.
$$

Subtracting the first equation from the second equation in (86), we obtain

$$
\begin{aligned}
& \partial_{t}\left(M_{2}-M_{1}\right)+\left(M_{2}-M_{1}\right)+\alpha_{-}\left(M_{2}-M_{1}\right) \\
& \quad+\alpha_{+}\left(M_{2}-M_{1}\right)+2 M_{3}=0,
\end{aligned}
$$

and we simplify the above computation by letting $M_{5}=M_{2}-M_{1}$.

So,

$$
\partial_{t} M_{5}+\left(1+\alpha_{+}+\alpha_{-}\right) M_{5}+2 M_{3}=0 .
$$

Thus,

$$
\left\{\begin{array}{l}
\partial_{t} M_{5}=-\left(1+\alpha_{+}+\alpha_{-}\right) M_{5}-2 M_{3}, \\
\partial_{t} M_{3}=i k \times M_{4}+M_{5}, \\
\partial_{t} M_{4}=-i k \times M_{3},
\end{array}\right.
$$

where we write the initial data in the form

$$
\left.\left[M_{3}, M_{4}, M_{5}\right]\right|_{t=0}=\left[M_{3,0}, M_{4,0}, M_{5,0}\right] \text {, }
$$

with

$$
\begin{aligned}
& M_{5,0}=-\tilde{k} \times\left(\widetilde{k} \times\left(\widehat{u}_{-}-\widehat{u}_{+}\right)\right), \\
& M_{3,0}=-\tilde{k} \times\left(\widetilde{k} \times \widehat{E}_{0}\right), \\
& M_{4,0}=-\tilde{k} \times\left(\tilde{k} \times \widehat{B}_{0}\right) .
\end{aligned}
$$

Taking the time derivative of $\partial_{t} M_{3}$ and substituting $\partial_{t} M_{5}$ and $\partial_{t} M_{4}$ as given in (89), we get that

$\partial_{t t} M_{3}=k \times\left(k \times M_{3}\right)-\left(1+\alpha_{+}+\alpha_{-}\right) M_{5}-2 M_{3}$.

From $k \cdot M_{2}=0$, we find $k \times\left(k \times M_{3}\right)=-|k|^{2} M_{3}$; thus,

$$
\partial_{t t} M_{3}+\left(2+|k|^{2}\right) M_{3}=-\left(1+\alpha_{+}+\alpha_{-}\right) M_{5} .
$$

Now, taking the time derivative of (93) and replacing $\partial_{t} M_{5}$ by the first equation of (89) and taking the sum with (93), we get

$$
\begin{gathered}
\partial_{t t t} M_{3}+\left(1+\alpha_{+}+\alpha_{-}\right) \partial_{t t} M_{3}+\left(2+|k|^{2}\right) \partial_{t} M_{3} \\
+\left(1+\alpha_{+}+\alpha_{-}\right)|k|^{2} M_{3}=0,
\end{gathered}
$$

with the initial data

$$
\left\{\begin{array}{l}
\left.M_{3}\right|_{t=0}=M_{3,0}, \\
\left.\partial_{t} M_{3}\right|_{t=0}=i k \times M_{4,0}+M_{5,0}, \\
\left.\partial_{t t} M_{3}\right|_{t=0}=-\left(1+\alpha_{+}+\alpha_{-}\right) M_{5,0}-\left(2+|k|^{2}\right) M_{3,0} .
\end{array}\right.
$$

Note that the characteristic equation of (94) is

$$
\begin{aligned}
F(x):= & \chi^{3}+\left(1+\alpha_{+}+\alpha_{-}\right) \chi^{2}+\left(2+|k|^{2}\right) \chi \\
& +\left(1+\alpha_{+}+\alpha_{-}\right)|k|^{2}=0 .
\end{aligned}
$$

Set

$$
\begin{aligned}
x_{1} & =M_{3}, \\
x_{2} & =\dot{x}_{1} \\
x_{3} & =\dot{x}_{2} \\
x & =\left[x_{1}, x_{2}, x_{3}\right]^{T} .
\end{aligned}
$$

Then, equation (94) is written as

$$
x_{t}=\Phi(k) x
$$

where

$$
\Phi(k)=\left[\begin{array}{ccc}
0 & 1 & 0 \\
0 & 0 & 1 \\
-\left(1+\alpha_{+}+\alpha_{-}\right)|k|^{2} & -\left(2+|k|^{2}\right) & -\left(1+\alpha_{+}+\alpha_{-}\right)
\end{array}\right] .
$$

Asymptotic Expansion of Eigenvalues. Let $\lambda_{j}(k)$ be the eigenvalues of the matrix $\Phi(k)$. We will find the asymptotic expansion of the eigenvalues $\lambda_{j}(k)$ for $|k| \longrightarrow 0$ and $|k| \longrightarrow \infty$.

The eigenvalues $\lambda_{j}(k), j=1,2,3$, are the solutions of the characteristic equation which can be written as

$$
\begin{aligned}
\operatorname{det}(\Phi(k)-\lambda I)= & \lambda^{3}+\left(1+\alpha_{+}+\alpha_{-}\right) \lambda^{2}+\left(2+|k|^{2}\right) \lambda \\
& +\left(1+\alpha_{+}+\alpha_{-}\right)|k|^{2}=0 .
\end{aligned}
$$

The eigenvector for $\Phi(k)$ is

$$
b(\lambda)=\left[\begin{array}{l}
1 \\
\lambda \\
\lambda^{2}
\end{array}\right] .
$$

(i) When $|k| \longrightarrow 0, \lambda_{j}(k)$ has the following asymptotic expansion:

$$
\lambda_{j}(k)=\lambda_{j}^{(0)}+\lambda_{j}^{(1)} k+\lambda_{j}^{(2)} k^{2}+\ldots,
$$

where each coefficient $\lambda_{j}^{(n)}$ is given by the direct computation as 


$$
\begin{aligned}
& \lambda_{1}^{(0)}=\lambda_{1}^{(1)} \\
& \lambda_{1}^{(2)}=-\frac{1}{2}\left(1+\alpha_{+}+\alpha_{-}\right), \\
& \lambda_{2}^{(0)}=\frac{-\left(1+\alpha_{+}+\alpha_{-}\right)+\sqrt{\left(1+\alpha_{+}+\alpha_{-}\right)^{2}-8}}{2}, \\
& \lambda_{2}^{(1)}=0, \\
& \lambda_{2}^{(2)}=\frac{-\left(1+\alpha_{+}+\alpha_{-}\right)-\sqrt{\left(1+\alpha_{+}+\alpha_{-}\right)^{2}-8}}{4}, \\
& \lambda_{3}^{(0)}=\frac{-\left(1+\alpha_{+}+\alpha_{-}\right)-\sqrt{\left(1+\alpha_{+}+\alpha_{-}\right)^{2}-8}}{2}, \\
& \lambda_{3}^{(2)}=\frac{-\left(1+\alpha_{+}+\alpha_{-}\right)+\sqrt{\left(1+\alpha_{+}+\alpha_{-}\right)^{2}-8}}{4} .
\end{aligned}
$$

Thus, the approximation of the eigenvalue when $|k| \longrightarrow 0$ is

$$
\begin{aligned}
& \lambda_{1}=-\frac{1}{2}\left(1+\alpha_{+}+\alpha_{-}\right) k^{2}, \\
& \lambda_{2}= \frac{-\left(1+\alpha_{+}+\alpha_{-}\right)+\sqrt{\left(1+\alpha_{+}+\alpha_{-}\right)^{2}-8}}{2} \\
& \lambda_{3}=-\left[\frac{\left(1+\alpha_{+}+\alpha_{-}\right)+\sqrt{\left(1+\alpha_{+}+\alpha_{-}\right)^{2}-8}}{4}\right] k^{2}, \\
&-\left[\frac{\left(1+\alpha_{+}+\alpha_{-}\right)-\sqrt{\left(1+\alpha_{+}+\alpha_{-}\right)^{2}-8}}{2}\right] \\
& 4
\end{aligned}
$$

Therefore, if we set

$$
D(t)=\left[\begin{array}{lll}
b\left(\lambda_{1}\right) e^{\lambda_{1} t} & b\left(\lambda_{2}\right) e^{\lambda_{2} t} & b\left(\lambda_{3}\right) e^{\lambda_{3} t}
\end{array}\right]
$$

the Green matrix $G$ for (98) is given by $G(t)=$ $D(t) D^{-1}(0)$, and the solution is represented as

$$
x=G(t) x_{0}
$$

where each component of this solution is itself a $3 \times 3$ diagonal matrix. We integrate the first and the third equations of (89), and we get the following expressions:

$$
\begin{aligned}
& M_{5}(t, k)=M_{5,0}(k) e^{-t\left(1+\alpha_{+}+\alpha_{-}\right)}+2 e^{-t\left(1+\alpha_{+}+\alpha_{-}\right)}\left[\int_{0}^{t} c_{1}(k) e^{\left(1+\alpha_{+}+\alpha_{-}+\eta\right) s} \mathrm{~d} s+\int_{0}^{t} e^{-t\left(1+\alpha_{+}+\alpha_{-}+\beta\right) s}\left(c_{2}(k) \cos \omega s+c_{3}(k) \sin \omega s\right) \mathrm{d} s\right], \\
& M_{4}(t, k)=M_{4,0}(k)-i k \times \int_{0}^{t} c_{1}(k) e^{\eta s}+e^{\beta s}\left(c_{2}(k) \cos \omega s+c_{3}(k) \sin \omega s\right) \mathrm{d} s,
\end{aligned}
$$

where

$$
\begin{aligned}
c_{1}= & \frac{1}{3 \eta^{2}+2\left(1+\alpha_{+}+\alpha_{-}\right) \eta+2+|k|^{2}}\left[-\eta \mathbf{I}_{3} M_{5,0}(t, k)+\eta\left(1+\alpha_{+}+\alpha_{-}+\eta\right) \mathbf{I}_{3} M_{3,0}(t, k)+\left(1+\alpha_{+}+\alpha_{-}+\eta\right) i k \times M_{4,0}(t, k)\right], \\
c_{2}= & \frac{1}{3 \eta^{2}+2\left(1+\alpha_{+}+\alpha_{-}\right) \eta+2+|k|^{2}}\left[\eta \mathbf{I}_{3} M_{5,0}(t, k)+\left(2 \eta+\eta\left(1+\alpha_{+}+\alpha_{-}+\eta\right)+|k|^{2}+2\right) \mathbf{I}_{3} M_{3,0}(t, k)-\left(1+\alpha_{+}+\alpha_{-}+\eta\right) i k \times M_{4,0}(t, k)\right], \\
c_{3}= & \frac{1}{3 \eta^{2}+2\left(1+\alpha_{+}+\alpha_{-}\right) \eta+2+|k|^{2}}\left[\frac{-(3 / 2) \eta^{2}-(3 / 2) \eta\left(1+\alpha_{+}+\alpha_{-}\right)-\left(2+|k|^{2}\right)}{\omega} \mathbf{I}_{3} M_{5,0}(t, k)\right. \\
& \left.+\frac{\left(1+\alpha_{+}+\alpha_{-}+\eta\right)\left(\eta\left(1+\alpha_{+}+\alpha_{-}\right)+2+|k|^{2}\right)}{2 \omega} \mathbf{I}_{3} M_{3,0}(t, k)+\frac{\left(-\left(1+\alpha_{+}+\alpha_{-}\right)^{2} / 2\right)+(3 / 2) \eta^{2}+\left(2+|k|^{2}\right)}{\omega} i k \times M_{4,0}(t, k)\right] .
\end{aligned}
$$


After a series of calculation using the symbolic manipulator and definition (84), the solution can be written as

$$
\begin{aligned}
\left(-\widetilde{k} \times\left(\widetilde{k} \times \widehat{u}_{-}\right)\right)-\left(-\widetilde{k} \times\left(\widetilde{k} \times \widehat{u}_{+}\right)\right)= & O(1) e^{-\theta k^{2} t}\left[\left(-\widetilde{k} \times\left(\widetilde{k} \times \widehat{u}_{-0}\right)\right)-\left(-\widetilde{k} \times\left(\widetilde{k} \times \widehat{u}_{+0}\right)\right)\right] \\
& +\left(O(1) e^{-\theta k^{2} t}-O(1) e^{-\theta t}\right)\left(-\widetilde{k} \times\left(\widetilde{k} \times \widehat{E}_{0}\right)\right)+\left(O(1) e^{-\theta k^{2} t}-O(1) e^{-\theta t}-O(1) i e^{-\theta t}\right) \\
& \cdot\left(-\widetilde{k} \times\left(\widetilde{k} \times \widehat{B}_{0}\right)\right), \\
-\widetilde{k} \times(\widetilde{k} \times \widehat{E})= & \left(O(1) e^{-\theta t}-O(1) i e^{-\theta t}\right)\left(-\widetilde{k} \times\left(\widetilde{k} \times \widehat{E}_{0}\right)\right)+\left(O(1) i e^{-\theta t}\right)\left(-\widetilde{k} \times\left(\widetilde{k} \times \widehat{B}_{0}\right)\right), \\
-\widetilde{k} \times(\widetilde{k} \times \widehat{B})= & \left(O(1) i e^{-\theta t}\right)\left(-\widetilde{k} \times\left(\widetilde{k} \times \widehat{E}_{0}\right)\right)+\left(O(1) e^{-\theta t}-O(1) i e^{-\theta t}\right)\left(-\widetilde{k} \times\left(\widetilde{k} \times \widehat{B}_{0}\right)\right) .
\end{aligned}
$$

By using (73) and substituting (74) in the first equation of (109), we obtain

$$
\begin{aligned}
\widehat{u}_{-}-\widehat{u}_{+}= & \frac{i \widetilde{k}}{|k|}\left(O(1) e^{-\theta t}+O(1) e^{-\theta k^{2} t} k^{2}\right) \widehat{\rho}_{+0}+\left(O(1) e^{-\theta t}+O(1) e^{-\theta k^{2} t} k^{2}\right) \tilde{k} \widetilde{k} \cdot \widehat{u}_{+0} \\
& +\frac{i \widetilde{k}}{|k|}\left(O(1) e^{-\theta t}+O(1) e^{-\theta k^{2} t} k^{2}\right) \widehat{\rho}_{-0}+\left(O(1) e^{-\theta t}+O(1) e^{-\theta k^{2} t} k^{2}\right) \widetilde{k} \widetilde{k} \cdot \widehat{u}_{-0} \\
& +O(1) e^{-\theta k^{2} t}\left[\left(-\widetilde{k} \times\left(\widetilde{k} \times \widehat{u}_{-0}\right)\right)-\left(-\tilde{k} \times\left(\widetilde{k} \times \widehat{u}_{+, 0}\right)\right)\right]+\left(O(1) e^{-\theta k^{2} t}-O(1) e^{-\theta t}\right)\left(-\widetilde{k} \times\left(\widetilde{k} \times \widehat{E}_{0}\right)\right) \\
& +\left(O(1) e^{-\theta k^{2} t}-O(1) e^{-\theta t}-O(1) i e^{-\theta t}\right)\left(-\widetilde{k} \times\left(\widetilde{k} \times \widehat{B}_{0}\right)\right) .
\end{aligned}
$$

Furthermore, we know from (51) that

$$
\nabla \cdot E=\rho_{+}-\rho_{-}
$$

and thus

$$
\widehat{E}=-\widetilde{k} \times(\widetilde{k} \times \widehat{E})-\frac{i \widetilde{k}}{|k|}\left(\widehat{\rho}_{+}-\widehat{\rho}_{-}\right) .
$$

Multiplying the first and the third equation of (72) by $\tilde{k}$ and substituting the result into equation (112), we obtain

$$
\begin{aligned}
\widehat{E}= & \frac{-i \widetilde{k}}{|k|}\left[\left(O(1) e^{-\theta t}+O(1) e^{-\theta k^{2} t}\right) \widehat{\rho}_{+0}-\left(O(1) e^{-\theta t}+O(1) e^{-\theta k^{2} t}\right) i k \cdot \widehat{u}_{+0}\right. \\
& \left.+\left(O(1) e^{-\theta t}+O(1) e^{-\theta k^{2} t}\right) \widehat{\rho}_{-0}-\left(O(1) e^{-\theta t}+O(1) e^{-\theta k^{2} t}\right) i k \cdot \widehat{u}_{-0}\right] \\
& +\left(O(1) e^{-\theta t}-O(1) i e^{-\theta t}\right)\left(-\widetilde{k} \times\left(\widetilde{k} \times \widehat{E}_{0}\right)\right)+O(1) i e^{-\theta t}\left(-\tilde{k} \times\left(\widetilde{k} \times \widehat{B}_{0}\right)\right) .
\end{aligned}
$$

Now, since $\tilde{k} \widetilde{k} \cdot \widehat{B}=0$, we have

$$
\widehat{B}=\left(O(1) i e^{-\theta t}\right)\left(-\widetilde{k} \times\left(\widetilde{k} \times \widehat{E}_{0}\right)\right)+\left(O(1) e^{-\theta t}-O(1) i e^{-\theta t}\right)\left(-\widetilde{k} \times\left(\widetilde{k} \times \widehat{B}_{0}\right)\right)
$$


Moreover, taking the sum of the first and the second equations of (86), we obtain

$\partial_{t}\left(M_{1}+M_{2}\right)+\left(M_{1}+M_{2}\right)+\left(M_{2}-M_{1}\right)\left(\alpha_{-}-\alpha_{+}\right)=0$.

Thus,

$$
\begin{aligned}
M_{1}+M_{2}= & e^{-\theta t}\left(M_{1,0}+M_{2,0}\right) \\
& \left.+\int_{0}^{t} e^{-(t-s)}\left(M_{2}-M_{1}\right)\left(\alpha_{-}-\alpha_{+}\right)\right) \mathrm{d} s .
\end{aligned}
$$

Substituting the first and the second equations of (84) into equation (116), we get that

$$
\begin{aligned}
& \left(-\tilde{k} \times\left(\tilde{k} \times \widehat{u}_{+}\right)\right)+\left(-\tilde{k} \times\left(\tilde{k} \times \widehat{u}_{-}\right)\right)=e^{-\theta t}\left[\left(-\tilde{k} \times\left(\widetilde{k} \times \widehat{u}_{+0}\right)\right)+\left(-\tilde{k} \times\left(\widetilde{k} \times \widehat{u}_{-0}\right)\right)\right] \\
& \quad+\int_{0}^{t} e^{-(t-s)}\left[\begin{array}{c}
O(1) e^{-\theta k^{2} s}\left[\left(-\widetilde{k} \times\left(\tilde{k} \times \widehat{u}_{-0}\right)\right)-\left(-\widetilde{k} \times\left(\widetilde{k} \times \widehat{u}_{+0}\right)\right)\right]+\left(O(1) e^{-\theta k^{2} s}-O(1) e^{-\theta s}\right)\left(-\widetilde{k} \times\left(\widetilde{k} \times \widehat{E}_{0}\right)\right) \\
+\left(O(1) e^{-\theta k^{2} s}-O(1) e^{-\theta s}-O(1) i e^{-\theta s}\right)\left(-\tilde{k} \times\left(\widetilde{k} \times \widehat{B}_{0}\right)\right)
\end{array}\right] \mathrm{d} s .
\end{aligned}
$$

Next, substituting (74) into the above computations, we obtain

$$
\begin{aligned}
\widehat{u}_{+}(t, k)+\widehat{u}_{-}(t, k)= & \frac{\tilde{k}}{|k|}\left(O(1) e^{-\theta t}+O(1) e^{-\theta k^{2} t} k^{2}\right) \widehat{\rho}_{+0}+\left(O(1) e^{-\theta t}+O(1) e^{-\theta k^{2} t} k^{2}\right) \tilde{k} \widetilde{k} \cdot \widehat{u}_{+0} \\
& +\frac{i \widetilde{k}}{|k|}\left(O(1) e^{-\theta t}+O(1) e^{-\theta k^{2} t} k^{2}\right) \widehat{\rho}_{-0}+\left(O(1) e^{-\theta t}+O(1) e^{-\theta k^{2} t} k^{2}\right) \widetilde{k} \widetilde{k} \cdot \widehat{u}_{-0} \\
& +e^{-\theta t}\left[\left(-\widetilde{k} \times\left(\widetilde{k} \times \widehat{u}_{+0}\right)\right)+\left(-\widetilde{k} \times\left(\widetilde{k} \times \widehat{u}_{-0}\right)\right)\right]+O(1)\left(e^{-\theta k^{2} t}-e^{-t}\right)\left[\left(-\widetilde{k} \times\left(\widetilde{k} \times \widehat{u}_{+0}\right)\right)-\left(-\widetilde{k} \times\left(\widetilde{k} \times \widehat{u}_{-0}\right)\right)\right] \\
& +O(1)\left(e^{-\theta k^{2} t}-e^{-t}\right)\left(-\widetilde{k} \times\left(\widetilde{k} \times \widehat{E}_{0}\right)\right)+O(1)\left(e^{-\theta t}-e^{-t}\right)\left(-\widetilde{k} \times\left(\widetilde{k} \times \widehat{E}_{0}\right)\right) \\
& +O(1)\left(e^{-\theta k^{2} t}-e^{-t}\right)\left(-\widetilde{k} \times\left(\widetilde{k} \times \widehat{B}_{0}\right)\right)-O(1)\left(e^{-\theta t}-e^{-t}\right)\left(-\widetilde{k} \times\left(\widetilde{k} \times \widehat{B}_{0}\right)\right)-O(1)\left(e^{-\theta t}-e^{-t}\right)\left(-\widetilde{k} \times i\left(\widetilde{k} \times \widehat{B}_{0}\right)\right) .
\end{aligned}
$$

Now, taking the sum and difference, respectively, of (110) and (118), we obtain

$$
\begin{aligned}
\widehat{u}_{ \pm}(t, k)= & \frac{i \widetilde{k}}{|k|}\left(O(1) e^{-\theta t}+O(1) e^{-\theta k^{2} t} k^{2}\right) \widehat{\rho}_{+0}+\left(O(1) e^{-\theta t}+O(1) e^{-\theta k^{2} t} k^{2}\right) \tilde{k} \widetilde{k} \cdot \widehat{u}_{+0} \\
& \left.+\frac{i \widetilde{k}}{|k|}(O(1)) e^{-\theta t}+O(1) e^{-\theta k^{2} t} k^{2}\right) \widehat{\rho}_{-0}+\left(O(1) e^{-\theta t}+O(1) e^{-\theta k^{2} t} k^{2}\right) \tilde{k} \widetilde{k} \cdot \widehat{u}_{-0} \\
& +O(1) e^{-\theta k^{2} t}\left(-\widetilde{k} \times\left(\widetilde{k} \times \widehat{u}_{-0}\right)\right)-\left(-\widetilde{k} \times\left(\widetilde{k} \times \widehat{u}_{+0}\right)\right)+\left(O(1) e^{-\theta k^{2} t}-O(1) e^{-\theta t}\right)\left(-\widetilde{k} \times\left(\widetilde{k} \times \widehat{E}_{0}\right)\right) \\
& +\left(O(1) e^{-\theta k^{2} t}-O(1) e^{-\theta t}-O(1) i e^{-\theta t}\right)\left(-\widetilde{k} \times\left(\widetilde{k} \times \widehat{B}_{0}\right)\right)+\frac{i \widetilde{k}}{|k|} O(1) e^{-\theta t} \widehat{\rho}_{+0}+O(1) e^{-\theta t} \widetilde{k} \widetilde{k} \cdot \widehat{u}_{+0}-\frac{i k}{|k|} O(1) e^{-\theta t} \widehat{\rho}_{-0} \\
& +O(1) e^{-\theta t} \widetilde{k} \widetilde{k} \cdot \widehat{u}_{-0}+e^{-\theta t}\left[\left(-\widetilde{k} \times\left(\widetilde{k} \times \widehat{u}_{+0}\right)\right)+\left(-\widetilde{k} \times\left(\widetilde{k} \times \widehat{u}_{-0}\right)\right)\right] \\
& +O(1) e^{-\theta k^{2} t}+O(1) e^{-\theta k^{2} t}-O(1) e^{-\theta t}+O(1) e^{-\theta k^{2} t}+O(1) e^{-\theta t}-O(1) i e^{-\theta t}-O(1) e^{-t}
\end{aligned}
$$

(ii) When $|k| \longrightarrow \infty, \lambda_{j}(k)$ has the following asymptotic $\quad \lambda_{j}(k)=\mu_{j}^{(1)} k+\mu_{j}^{(0)}+\mu_{j}^{(-1)} k^{-1}+\mu_{j}^{(-2)} k^{-2}+\ldots$ expansion: 
Each coefficient $\mu_{j}^{(n)}$ is given by direct computation as

$$
\begin{aligned}
& \mu_{1}^{(1)}=0, \\
& \mu_{1}^{(0)}=-\left(1+\alpha_{+}+\alpha_{-}\right), \\
& \mu_{2}^{(1)}=i, \\
& \mu_{2}^{(0)}=0, \\
& \mu_{2}^{(-1)}=\frac{-2}{3 i}, \\
& \mu_{2}^{(-2)}=-\frac{4}{9} \\
& \mu_{3}^{(1)}=-i, \\
& \mu_{3}^{(0)}=0, \\
& \mu_{3}^{(-1)}=\frac{2}{3 i}, \\
& \mu_{3}^{(-2)}=\frac{4}{9} .
\end{aligned}
$$

The approximation of the eigenvalue when $|k| \longrightarrow \infty$ is

$$
\begin{aligned}
& \lambda_{1}=-\left(1+\alpha_{+}+\alpha_{-}\right), \\
& \lambda_{2}=i k-\frac{2}{3 i} k^{-1}-\frac{4}{9} k^{-2}, \\
& \lambda_{3}=-i k+\frac{2}{3 i} k^{-1}-\frac{4}{9} k^{-2} .
\end{aligned}
$$

Hence, the Green matrix $G$ for (98) is given by $G(t)=D(t) D^{-1}(0)$, and the solution is represented as

$$
x=G(t) x_{0}
$$

where each component of this solution is itself a $3 \times 3$ diagonal matrix. After a series of calculation using the symbolic manipulator and equation (84), the solution can be written as

$$
\begin{aligned}
& \left(-\widetilde{k} \times\left(\widetilde{k} \times \widehat{u}_{-}\right)\right)-\left(-\widetilde{k} \times\left(\widetilde{k} \times \widehat{u}_{+}\right)\right)=O(1) e^{-\theta t}\left[\left(-\widetilde{k} \times\left(\widetilde{k} \times \widehat{u}_{-, 0}\right)\right)-\left(-\widetilde{k} \times\left(\widetilde{k} \times \widehat{u}_{+0}\right)\right)\right] \\
& +\frac{O(1)}{|k|} e^{-\theta k^{-2} t}\left(-\tilde{k} \times\left(\widetilde{k} \times \widehat{E}_{0}\right)\right)+\frac{O(1)}{|k|^{2}} e^{-\theta t}-\frac{O(1)}{|k|^{2}} e^{-\theta k^{-2} t}\left(-\tilde{k} \times\left(\widetilde{k} \times \widehat{B}_{0}\right)\right), \\
& -\tilde{k} \times(\widetilde{k} \times \widehat{E})=-O(1) e^{-\theta t}+O(1) e^{-\theta k^{-2} t}\left[\left(-\tilde{k} \times\left(\widetilde{k} \times \widehat{u}_{-0}\right)\right)-\left(-\tilde{k} \times\left(\widetilde{k} \times \widehat{u}_{+0}\right)\right)\right] \\
& +O(1)^{-\theta k^{-2} t}\left(-\tilde{k} \times\left(\widetilde{k} \times \widehat{E}_{0}\right)\right)+\frac{O(1)}{|k|} e^{-\theta k^{-2} t}\left(-\widetilde{k} \times\left(\widetilde{k} \times \widehat{B}_{0}\right)\right) \\
& -\tilde{k} \times(\widetilde{k} \times \widehat{B})=O(1) i|k| e^{-\theta k^{-2} t}\left[\left(-\widetilde{k} \times\left(\widetilde{k} \times \widehat{u}_{-0}\right)\right)-\left(-\tilde{k} \times\left(\widetilde{k} \times \widehat{u}_{+0}\right)\right)\right] \\
& -O(1) i|k| e^{-\theta t}\left(-\widetilde{k} \times\left(\widetilde{k} \times \widehat{E}_{0}\right)\right)+O(1) e^{-\theta k^{-2} t}\left(-\tilde{k} \times\left(\widetilde{k} \times \widehat{B}_{0}\right)\right) .
\end{aligned}
$$

By using (73) and substituting (80) into the first equation of the above computation, we obtain

$$
\begin{aligned}
\widehat{u}_{-}-\widehat{u}_{+}= & \left(O(1) e^{-\theta t} \tilde{k} \tilde{k}-O(1) e^{-\theta t} \frac{i \widetilde{k}}{|k|} i\right) \widehat{\rho}_{+0}+\left(O(1) e^{-\theta t}-O(1) e^{-\theta t} \frac{1}{|k|}\right) \tilde{k} \widetilde{k} \cdot \widehat{u}_{+0} \\
& +\left(O(1) e^{-\theta t} \frac{i \tilde{k}}{|k|}-O(1) e^{-\theta t} i \tilde{k} \tilde{k}\right) \widehat{\rho}_{-0}+\left(O(1) e^{-\theta t} \frac{1}{|k|}-O(1) e^{-\theta t}\right) \tilde{k} \widetilde{k} \cdot \widehat{u}_{-0} \\
& +O(1) e^{-\theta t}\left[\left(-\widetilde{k} \times\left(\widetilde{k} \times \widehat{u}_{-0}\right)\right)-\left(-\widetilde{k} \times\left(\widetilde{k} \times \widehat{u}_{+, 0}\right)\right)\right] \\
& +\frac{O(1)}{|k|} e^{-\theta k^{-2} t}\left(-\tilde{k} \times\left(\widetilde{k} \times \widehat{E}_{0}\right)\right)+\left(\frac{O(1)}{|k|^{2}} e^{-\theta t}-\frac{O(1)}{|k|^{2}} e^{-\theta k^{-2} t}\right)\left(-\tilde{k} \times\left(\widetilde{k} \times \widehat{B}_{0}\right)\right) .
\end{aligned}
$$


Furthermore, we have

$$
\widehat{E}=-\tilde{k} \times(\widetilde{k} \times \widehat{E})-\frac{i \widetilde{k}}{|k|}\left(\widehat{\rho}_{+}-\widehat{\rho}_{-}\right) .
$$

Therefore, multiplying the first and the third equation of (79) by $\widetilde{k}$ and substituting into the above equation, we obtain

$$
\begin{aligned}
\widehat{E}= & -\left[\left(O(1)-O(1)|k|^{-1}\right) e^{-\theta t} \widehat{\rho}_{+0}-\left(O(1)|k|^{-1}-O(1)\right) e^{-\theta t} i k \cdot \widehat{u}_{+0}\right. \\
& \left.+\left(O(1)|k|^{-1}-O(1)\right) e^{-\theta t} \widehat{\rho}_{-0}-\left(O(1)+O(1)|k|^{-1}\right) e^{-\theta t} i k \cdot \widehat{u}_{-0}\right] \\
& +\left(-O(1) e^{-\theta t}+O(1) e^{-\theta k^{-2} t}\right)\left[\left(-\widetilde{k} \times\left(\widetilde{k} \times \widehat{u}_{-0}\right)\right)-\left(-\widetilde{k} \times\left(\widetilde{k} \times \widehat{u}_{+, 0}\right)\right)\right] \\
& +O(1) e^{-\theta k^{-2} t}\left(-\widetilde{k} \times\left(\widetilde{k} \times \widehat{E}_{0}\right)\right)+\frac{O(1)}{|k|} e^{-\theta k^{-2} t}\left(-\widetilde{k} \times\left(\widetilde{k} \times \widehat{B}_{0}\right)\right) .
\end{aligned}
$$

Since $\tilde{k} \widetilde{k} \cdot \widehat{B}=0$, we get

$$
\begin{aligned}
\widehat{B}= & O(1) i|k| e^{-\theta k^{-2} t}\left(-\tilde{k} \times\left(\widetilde{k} \times \widehat{u}_{-, 0}\right)\right)-\left(-\widetilde{k} \times\left(\widetilde{k} \times \widehat{u}_{+, 0}\right)\right) \\
& -O(1) i|k| e^{-\theta t}\left(-\widetilde{k} \times\left(\widetilde{k} \times \widehat{E}_{0}\right)\right)+O(1) e^{-\theta k^{-2} t}\left(-\widetilde{k} \times\left(\widetilde{k} \times \widehat{B}_{0}\right)\right) .
\end{aligned}
$$

Substituting the first equation of (124) into equation (116) results in

$$
\begin{aligned}
\left(-\widetilde{k} \times\left(\widetilde{k} \times \widehat{u}_{+}\right)\right)+\left(-\widetilde{k} \times\left(\widetilde{k} \times \widehat{u}_{-}\right)\right)= & e^{-\theta t}\left[\left(-\widetilde{k} \times\left(\widetilde{k} \times \widehat{u}_{+0}\right)\right)+\left(-\widetilde{k} \times\left(\widetilde{k} \times \widehat{u}_{-0}\right)\right)\right] \\
& +\int_{0}^{t} e^{-(t-s)}\left[O(1) e^{-\theta s}\left(-\widetilde{k} \times\left(\widetilde{k} \times \widehat{u}_{-0}\right)\right)-\left(-\widetilde{k} \times\left(\widetilde{k} \times \widehat{u}_{+0}\right)\right)\right. \\
& \left.+\frac{O(1)}{|k|} e^{-\theta k^{-2} s}\left(-\widetilde{k} \times\left(\widetilde{k} \times \widehat{E}_{0}\right)\right)+\left(\frac{O(1)}{|k|^{2}} e^{-\theta s}-\frac{O(1)}{|k|^{2}} e^{-\theta k^{-2} s}\right)\left(-\widetilde{k} \times\left(\widetilde{k} \times \widehat{B}_{0}\right)\right)\right] \mathrm{d} s .
\end{aligned}
$$
obtain

Next, substituting equation (80) into equation (129), we 


$$
\begin{aligned}
& \widehat{u}_{+}(t, k)+\widehat{u}_{-}(t, k)=\left(O(1) e^{-\theta t} \widetilde{k} \tilde{k}-O(1) e^{-\theta t} \frac{i \widetilde{k}}{|k|} i\right) \widehat{\rho}_{+0}+\left(O(1) e^{-\theta t}-O(1) e^{-\theta t} \frac{1}{|k|}\right) \tilde{k} \tilde{k} \cdot \widehat{u}_{+0} \\
& +\left(O(1) e^{-\theta t} \frac{i \tilde{k}}{|k|}-O(1) e^{-\theta t} i \tilde{k} \tilde{k}\right) \widehat{\rho}_{-0}+\left(O(1) e^{-\theta t} \frac{1}{|k|}-O(1) e^{-\theta t}\right) \tilde{k} \tilde{k} \cdot \widehat{u}_{-0} \\
& +e^{-\theta t}\left[\left(-\tilde{k} \times\left(\widetilde{k} \times \widehat{u}_{+0}\right)\right)+\left(-\tilde{k} \times\left(\widetilde{k} \times \widehat{u}_{-0}\right)\right)\right]+O(1)\left(e^{-\theta t}-e^{-t}\right)\left[\left(-\tilde{k} \times\left(\tilde{k} \times \widehat{u}_{+0}\right)\right)+\left(-\tilde{k} \times\left(\widetilde{k} \times \widehat{u}_{-0}\right)\right)\right] \\
& +O(1)\left(e^{-\theta k^{-2} t}-e^{-t}\right)\left(-\tilde{k} \times\left(\tilde{k} \times \widehat{E}_{0}\right)\right)+O(1)\left(e^{-\theta t}-e^{-t}\right)\left(-\tilde{k} \times\left(\widetilde{k} \times \widehat{B}_{0}\right)\right)+O(1) \\
& \cdot\left(e^{-\theta k^{-2} t}-e^{-t}\right)\left(-\widetilde{k} \times\left(\widetilde{k} \times \widehat{B}_{0}\right)\right)
\end{aligned}
$$

Now, taking the sum and difference, respectively, of equations (125) and (130), we obtain

$$
\begin{aligned}
& \widehat{u}_{ \pm}(t, k)=\left(O(1) e^{-\theta t} \widetilde{k} \widetilde{k}-O(1) e^{-\theta t} \frac{i \widetilde{k}}{|k|} i\right) \widehat{\rho}_{+0}+\left(O(1) e^{-\theta t}-O(1) e^{-\theta t} \frac{1}{|k|}\right) \widetilde{k} \widetilde{k} \cdot \widehat{u}_{+0} \\
& +\left(O(1) e^{-\theta t} \frac{i \tilde{k}}{|k|}-O(1) e^{-\theta t} i \tilde{k} \tilde{k}\right) \widehat{\rho}_{-0}+\left(O(1) e^{-\theta t} \frac{1}{|k|}-O(1) e^{-\theta t}\right) \tilde{k} \widetilde{k} \cdot \widehat{u}_{-0} x \\
& +O(1) e^{-\theta t}\left[\left(-\widetilde{k} \times\left(\widetilde{k} \times \widehat{u}_{+0}\right)\right)+\left(-\tilde{k} \times\left(\widetilde{k} \times \widehat{u}_{-0}\right)\right)\right]+\frac{O(1)}{|k|} e^{-\theta k^{-2} t}\left(-\widetilde{k} \times\left(\widetilde{k} \times \widehat{E}_{0}\right)\right)+\left(\frac{O(1)}{|k|^{2}} e^{-\theta t}-\frac{O(1)}{|k|^{2}} e^{-\theta k^{-2} t}\right)\left(-\widetilde{k} \times\left(\widetilde{k} \times \widehat{B}_{0}\right)\right) \\
& +\frac{i \widetilde{k}}{|k|^{2}} O(1) e^{-\theta t} \widehat{\rho}_{+0}+\frac{O(1)}{|k|} e^{-\theta t} \tilde{k} \widetilde{k} \cdot \widehat{u}_{+0}-i \widetilde{k} O(1) e^{-\theta t} \widehat{\rho}_{-0}+O(1) e^{-\theta t} \tilde{k} \widetilde{k} \cdot \widehat{u}_{-0}+e^{-\theta t}\left[\left(-\widetilde{k} \times\left(\widetilde{k} \times \widehat{u}_{+0}\right)\right)+\left(-\widetilde{k} \times\left(\tilde{k} \times \widehat{u}_{-0}\right)\right)\right] \\
& +O(1) e^{-\theta t}+O(1) e^{-\theta k^{-2} t}+O(1) e^{-\theta t}-O(1) e^{-\theta k^{-2} t}-O(1) e^{-t} .
\end{aligned}
$$

Theorem 4. Let $1 \leq p, r \leq 2 \leq q \leq \infty$, and $l \geq 0$ and let $m \geq 0$ be an integer. Define

$$
\left[l+3\left(\frac{1}{r}-\frac{1}{q}\right)\right]_{+}= \begin{cases}{\left[l+3\left(\frac{1}{r}-\frac{1}{q}\right)\right]_{-}+1,} & \text { when } r \neq 2 \text { or } q \neq 2 \text { or } l \text { is not an integer } \\ l, & \text { when } r=q=2 \text { and } l \text { is an integer }\end{cases}
$$

where $[\cdot]_{-}$denotes the integer part of the argument. Suppose $U_{0}$ satisfies (53). Then, for any $t \geq 0, \nabla^{m} e^{t L} U_{0}$ satisfies the following time decay property:

$$
\begin{aligned}
\left\|\nabla^{m} e^{t L} U_{0}\right\|_{L^{q}} \leq & C(1+t)^{-(3 / 2)(1 / p-t 1 / q)-(m / 2)} \\
& \cdot\left\|U_{0}\right\|_{L^{p}}+C(1+t)^{-l / 2}\left\|\nabla^{m+[l+3(1 / r-t 1 / q)]_{+}} U_{0}\right\|_{L^{r}},
\end{aligned}
$$

where $C=C(p, q, r, l, m)$.

Theorem 5. Let $1 \leq p, r \leq 2 \leq q \leq \infty$, and $l \geq 0$ and let $m \geq 0$ be an integer. Assume $U(t)=e^{t L} U_{0}$ is the solution of the initial value problems (51) and (52) with initial data $\left[\rho_{ \pm 0}, u_{ \pm 0}, E_{0}, B_{0}\right]$ which satisfies (53). Then, for any $t \geq 0, U=$ $\left[\rho_{ \pm}, u_{ \pm}, E, B\right]$ satisfies 


$$
\begin{aligned}
& \left\|\nabla^{m} \rho_{ \pm}(t)\right\|_{L^{q}} \leq C(1+t)^{-(3 / 2)(1 / p-1 / q)-(m / 2)}\left\|\left[\widehat{\rho}_{ \pm 0}, \widehat{u}_{ \pm, 0}\right]\right\|_{L^{p}}+C\left\|\left[\rho_{ \pm 0}, u_{ \pm 0}\right]\right\|_{L^{r}}, \\
& \left\|\nabla^{m} u_{ \pm}(t)\right\|_{L^{q}} \leq C e^{-t / 2}\left(\left\|\rho_{ \pm 0}\right\|_{L^{p}}+\left\|\nabla^{m+[3(1 / r-1 / q)]_{+}}\left[\rho_{ \pm 0}, u_{ \pm 0}\right]\right\|_{L^{r}}\right) \\
& +C(1+t)^{-(3 / 2)(1 / p-1 / q)-((m+1) / 2)}\left\|\left[u_{ \pm 0}, E_{0}, B_{0}\right]\right\|_{L^{p}}+C(1+t)^{-(l+1) / 2}\left\|\nabla^{m+[l+3(1 / r-1 / q)]_{+}}\left[u_{ \pm 0}, E_{0}, B_{0}\right]\right\|_{L^{r}}, \\
& \left\|\nabla^{m} E(t)\right\|_{L^{q}} \leq C(1+t)^{-(3 / 2)(1 / p-1 / q)-((m+1) / 2)}\left\|\left[u_{ \pm 0}, E_{0}, B_{0}\right]\right\|_{L^{p}}+C(1+t)^{-l / 2}\left\|\nabla^{m+[l+3(1 / r-1 / q)]_{+}}\left[u_{ \pm 0}, E_{0}, B_{0}\right]\right\|_{L^{r}}, \\
& \left\|\nabla^{m} B(t)\right\|_{L^{q}} \leq C(1+t)^{-(3 / 2)(1 / p-1 / q)-((m) / 2)}\left\|\left[u_{ \pm 0}, E_{0}, B_{0}\right]\right\|_{L^{p}}+C(1+t)^{-l / 2}\left\|\nabla^{m+[l+3(1 / r-1 / q)]_{+}}\left[u_{ \pm 0}, E_{0}, B_{0}\right]\right\|_{L^{r}}
\end{aligned}
$$

where $C=C(p, q, r, l, m)$ and $[l+3(1 / r-1 / q)]_{+}$is defined in (132).

Proof. Let $1 \leq p, r \leq 2 \leq q \leq \infty$, and $m$ be a nonnegative integer. Let $q^{\prime}$ satisfy $1 / q+1 / q=1$. Using the Hausdorff-Young inequality, we prove (134) as follows:

$$
\left\|\nabla^{m} \rho_{ \pm}(t, k)\right\|_{L_{x}^{q}} \leq C\left\||k|^{m} \widehat{\rho}_{ \pm}\right\|_{L^{q^{\prime}}(|k| \leq 1)}+C\left\||k|^{m} \widehat{\rho}_{ \pm}\right\|_{L^{q^{\prime}}(|k| \geq 1)^{\circ}}
$$

We estimate the first term of (138) using the Hölder inequality $1 / q^{\prime}=1 / p^{\prime}+\left(p^{\prime}-q^{\prime}\right) / p^{\prime} q^{\prime}$ with $1 / p^{\prime}+1 / p=1$ and fixing $\epsilon>0$ sufficiently small; that is,

$$
\begin{aligned}
& \left\||k|^{m}\left[\widehat{\rho}_{ \pm 0}, \widehat{u}_{ \pm 0}\right]\left[e^{-\theta t}+e^{-\theta|k|^{2} t}\right]\right\|_{L^{\prime}(|k| \leq 1)} \\
& \quad=\left\||k|^{-\left(p^{\prime}-q^{\prime}\right) / p^{\prime} q^{\prime}(3-\varepsilon)}|k|^{m+\left(\left(p^{\prime}-q^{\prime}\right) / p^{\prime} q^{\prime}\right)(3-\varepsilon)}\left[\widehat{\rho}_{ \pm 0}, \widehat{u}_{ \pm 0}\right]\left[e^{-\theta t}+e^{-\theta|k|^{2} t}\right]\right\|_{L^{q^{\prime}}(|k| \leq 1)} \\
& \quad \leq\left\||k|^{-(3-\varepsilon)}\right\|^{-\left(p^{\prime}-q^{\prime}\right) / p^{\prime} q^{\prime}}\left\||k|^{m+\left(p^{\prime}-q^{\prime}\right) / p^{\prime} q^{\prime}}\left[\widehat{\rho}_{ \pm 0}, \widehat{u}_{ \pm 0}\right]\left[e^{-\theta t}+e^{-\theta|k|^{2} t}\right]\right\|_{L^{\prime}(|k| \leq 1)} \\
& \quad \leq\left\||k|^{m+\left(\left(p^{\prime}-q^{\prime}\right) / p^{\prime} q^{\prime}\right)(3-\varepsilon)}\left[\widehat{\rho}_{ \pm 0}, \widehat{u}_{ \pm 0}\right]\left[e^{-\theta t}+e^{-\theta|k|^{2} t}\right]\right\|_{L^{p^{\prime}}(|k| \leq 1)} \\
& \quad \leq C e^{-\theta t}\left\|\left[\widehat{\rho}_{ \pm 0}, \widehat{u}_{ \pm 0}\right]\right\|_{L^{p^{\prime}}(|k| \leq 1)}+C(t+1)^{-(3 / 2)\left(1 / q^{\prime}-1 / p^{\prime}\right)-(m+1 / 2)}\left\|\left[\widehat{\rho}_{ \pm 0}, \widehat{u}_{ \pm 0}\right]\right\|_{L^{p^{\prime}}(|k| \leq 1)} \\
& \quad \leq C e^{-\theta t}\left\|\left[\rho_{ \pm 0}, u_{ \pm 0}\right]\right\|_{L^{p}(|k| \leq 1)}+C(t+1)^{-3 / 2(1 / p-1 / q)-(m+1 / 2)}\left\|\left[\rho_{ \pm 0}, u_{ \pm 0}\right]\right\|_{L^{p}(|k| \leq 1)}
\end{aligned}
$$

Now, we estimate the second term; by taking $\epsilon>0$ sufficiently small and using the Hölder inequality $1 / q^{\prime}=$ $1 / r^{\prime}+\left(r^{\prime}-q^{\prime} / r^{\prime} q^{\prime}\right)$ with $1 / r^{\prime}+1 / r=1$, we find

$$
\begin{aligned}
& \left\||k|^{m}\left[\widehat{\rho}_{ \pm 0}, \widehat{u}_{ \pm 0}\right]\left[e^{-\theta t}+e^{-\theta|k|^{2} t}\right]\right\|_{L^{q^{\prime}}(|k| \geq 1)} \\
& \quad=\left\||k|^{-\left(r^{\prime}-q^{\prime} / r^{\prime} q^{\prime}\right)(3+\varepsilon)}|k|^{m+\left(p^{\prime}-q^{\prime} \mid p^{\prime} q^{\prime}\right)(3+\varepsilon)}\left[\widehat{\rho}_{ \pm 0}, \widehat{u}_{ \pm 0}\right] e^{-\theta t}\right\|_{L^{q^{\prime}}(|k| \geq 1)} \\
& \leq\left\||k|^{-(3+\varepsilon)}\right\|^{-\left(r^{\prime}-q^{\prime} / r^{\prime} q^{\prime}\right)}\left\||k|^{m+\left(r^{\prime}-q^{\prime} \mid r^{\prime} q^{\prime}\right)(3+\varepsilon)}\left[\widehat{\rho}_{ \pm 0}, \widehat{u}_{ \pm 0}\right] e^{-\theta t}\right\|_{L^{r^{\prime}}(|k| \geq 1)} \\
& \leq C\left\||k|^{m+\left(1 / q^{\prime}-1 / r^{\prime}\right)(3+\varepsilon)}\left[\widehat{\rho}_{ \pm 0}, \widehat{u}_{ \pm 0}\right] e^{-\theta t}\right\|_{L^{\prime}(|k| \geq 1)} \\
& \leq C e^{-\theta t}\left\|\nabla^{m+[3(1 / r-1 / q)]_{+}}\left[\rho_{ \pm 0}, u_{ \pm 0}\right]\right\|_{L^{\prime}(|k| \geq 1)}
\end{aligned}
$$

We prove (135) similarly. That is, $\left\|\nabla^{m} u_{ \pm}(t)\right\|_{L_{x}^{q}} \leq C\left\||k|^{m} \widehat{u}_{ \pm}\right\|_{L^{q^{\prime}}(|k| \leq 1)}+C\left\||k|^{m} \widehat{u}_{ \pm}\right\|_{L^{q^{\prime}}(|k| \geq 1)^{\prime}}$

$$
\begin{aligned}
\left\||k|^{m} \widehat{u}_{ \pm}(t, k)\right\|_{L^{q^{\prime}}(|k| \leq 1)}= & \left\||k|^{m} e^{-t / 2}\left[\widehat{\rho}_{ \pm 0}, \widehat{u}_{ \pm 0}, \widehat{E}_{0}\right]\right\|_{L^{q^{\prime}}} \\
& +\left\||k|^{m} e^{-\theta t}\left[\widehat{\rho}_{ \pm 0}, \widehat{u}_{ \pm 0}, \widehat{E}_{0}, \widehat{B}_{0}\right]\right\|_{L^{\prime}} \\
& +\left\||k|^{m} e^{-\theta|k|^{2} t}\left[\widehat{\rho}_{ \pm 0}, \widehat{u}_{ \pm 0}, \widehat{E}_{0}, \widehat{B}_{0}\right]\right\|_{L^{q^{\prime}}}
\end{aligned}
$$

where 
We estimate of the first term of (142) using the Hölder inequality $1 / q^{\prime}=1 / p^{\prime}+\left(p^{\prime}-q^{\prime}\right) / p^{\prime} q^{\prime}$ with $1 / p^{\prime}+1 / p=1$ and fixing $\epsilon>0$ sufficiently small:

$$
\begin{aligned}
& \left\||k|^{m} e^{-t / 2}\left[\widehat{\rho}_{ \pm 0}, \widehat{u}_{ \pm 0}, \widehat{E}_{0}\right]\right\|_{L^{q^{\prime}}}=\left(\left.\int_{\mathbb{R}^{3}}|k|^{m} e^{-t / 2}\left[\widehat{\rho}_{ \pm 0}, \widehat{u}_{ \pm 0}, \widehat{E}_{0}\right]\right|^{q^{\prime}}\right)^{1 / q^{\prime}} \\
& =\left(\left.\left.\int_{\mathbb{R}^{3}}|| k\right|^{-\left(p^{\prime}-q^{\prime} \mid p^{\prime} q^{\prime}\right)(3-\varepsilon)}|k|^{m+\left(p^{\prime}-q^{\prime} \mid p^{\prime} q^{\prime}\right)(3-\varepsilon)} e^{-t / 2}\left[\widehat{\rho}_{ \pm 0}, \widehat{u}_{ \pm 0}, \widehat{E}_{0}\right]\right|^{q^{\prime}}\right)^{1 / q^{\prime}} \\
& \leq e^{-t / 2}\left\||k|^{-(3-\varepsilon)}\right\|^{-\left(p^{\prime}-q^{\prime} \mid p^{\prime} q^{\prime}\right)}\left\||k|^{m+\left(p^{\prime}-q^{\prime} \mid p^{\prime} q^{\prime}\right)}\left[\widehat{\rho}_{ \pm 0}\right]\right\|_{L^{p^{\prime}}(|k| \leq 1)} \\
& \leq e^{-t / 2}\left\||k|^{m+\left(p^{\prime}-q^{\prime} \mid p^{\prime} q^{\prime}\right)(3-\varepsilon)}\left[\widehat{\rho}_{ \pm 0}\right]\right\|_{L^{p^{\prime}}(|k| \leq 1)} \leq C e^{-t / 2}\left\|\left[\widehat{\rho}_{ \pm 0}\right]\right\|_{L^{p^{\prime}}(|k| \leq 1)} \\
& \leq C e^{-t / 2}\left\|\left[\rho_{ \pm 0}\right]\right\|_{L^{p}(|k| \leq 1)} \text {. }
\end{aligned}
$$

We estimate the second term of (142) by

$$
\begin{aligned}
\left\||k|^{m} e^{-\theta|k|^{2} t}\left[\widehat{\rho}_{ \pm 0}, \widehat{u}_{ \pm 0}, \widehat{E}_{0}, \widehat{B}_{0}\right]\right\|_{L^{q^{\prime}}} & =\left(\left.\left.\int_{\mathbb{R}^{3}}|| k\right|^{m+1} e^{-\theta|k|^{2} t}\left[\widehat{u}_{ \pm 0}, \widehat{E}_{0}, \widehat{B}_{0}\right]\right|^{q^{\prime}}\right)^{1 / q^{\prime}} \\
& =\left(\left.\left.\int_{\mathbb{R}^{3}}|| k\right|^{-\left(p^{\prime}-q^{\prime} \mid p^{\prime} q^{\prime}\right)(3-\varepsilon)}|k|^{m+\left(p^{\prime}-q^{\prime} \mid p^{\prime} q^{\prime}\right)(3-\varepsilon)} e^{-\theta|k|^{2} t}\left[\widehat{u}_{ \pm 0}, \widehat{E}_{0}, \widehat{B}_{0}\right]\right|^{q^{\prime}}\right)^{1 / q^{\prime}} \\
& \leq\left\||k|^{-(3-\varepsilon)}\right\|^{-\left(p^{\prime}-q^{\prime} \mid p^{\prime} q^{\prime}\right)}\left\||k|^{m+\left(p^{\prime}-q^{\prime} \mid p^{\prime} q^{\prime}\right)} e^{-\theta|k|^{2} t}\left[\widehat{u}_{ \pm 0}, \widehat{E}_{0}, \widehat{B}_{0}\right]\right\|_{L^{p^{\prime}}(|k| \leq 1)} \\
& \leq e^{-t / 2}\left\||k|^{m+\left(p^{\prime}-q^{\prime} \mid p^{\prime} q^{\prime}\right)(3-\varepsilon)}\left[\widehat{\rho}_{ \pm 0}\right]\right\|_{L^{p^{\prime}}(|k| \leq 1)} \leq C e^{-t / 2}\left\|\left[\widehat{\rho}_{ \pm 0}\right]\right\|_{L^{p^{\prime}}(|k| \leq 1)} \\
& \leq C e^{-t / 2}\left\|\left[\rho_{ \pm 0}\right]\right\|_{L^{p}(|k| \leq 1)}
\end{aligned}
$$

We estimate the third term of (142) in two parts. First, where take $\epsilon>0$ sufficiently small and use the Hölder inequality $1 / q^{\prime}=1 / r^{\prime}+\left(r^{\prime}-q^{\prime} / r^{\prime} q^{\prime}\right)$ with $1 / r^{\prime}+1 / r=1$ :

$\left\||k|^{m} \widehat{u}_{ \pm}(t, k)\right\|_{L^{\prime}(|k| \geq 1)}=\left\||k|^{m} e^{-t / 2}\left[\widehat{\rho}_{ \pm 0}, \widehat{u}_{ \pm 0}, \widehat{E}_{0}\right]\right\|_{L^{q^{\prime}}}$

$$
\begin{aligned}
& +\left\||k|^{m} e^{-\theta t}\left[\widehat{\rho}_{ \pm 0}, \widehat{u}_{ \pm 0}, \widehat{E}_{0}, \widehat{B}_{0}\right]\right\|_{L^{q^{\prime}}} \\
& +\left\||k|^{m} \frac{1}{|k|} e^{-\theta|k|^{2} t}\left[\widehat{\rho}_{ \pm 0}, \widehat{u}_{ \pm 0}, \widehat{E}_{0}, \widehat{B}_{0}\right]\right\|_{L^{q^{\prime}}}
\end{aligned}
$$

$$
\begin{aligned}
& \left\||k|^{m} e^{-t / 2}\left[\widehat{\rho}_{ \pm 0}, \widehat{u}_{ \pm 0}, \widehat{E}_{0}\right]\right\|_{L^{q^{\prime}}}=\left(\left.\int_{\mathbb{R}^{3}}|k|^{m} e^{-t / 2}\left[\widehat{\rho}_{ \pm 0}, \widehat{u}_{ \pm 0}, \widehat{E}_{0}\right]\right|^{q^{\prime}}\right)^{1 / q^{\prime}} \\
& =\left(\left.\left.\int_{\mathbb{R}^{3}}|| k\right|^{-\left(r^{\prime}-q^{\prime} / r^{\prime} q^{\prime}\right)(3+\varepsilon)}|k|^{m+\left(r^{\prime}-q^{\prime} / r^{\prime} q^{\prime}\right)(3+\varepsilon)} e^{-t / 2}\left[\widehat{\rho}_{ \pm 0}, \widehat{u}_{ \pm 0}, \widehat{E}_{0}\right]\right|^{q^{\prime}}\right)^{1 / q^{\prime}} \\
& \leq e^{-t / 2}\left\||k|^{-(3+\varepsilon)}\right\|^{-\left(r^{\prime}-q^{\prime} / r^{\prime} q^{\prime}\right)}\left\||k|^{m+\left(r^{\prime}-q^{\prime} \mid r^{\prime} q^{\prime}\right)(3+\varepsilon)}\left[\widehat{\rho}_{ \pm 0}\right]\right\|_{L^{p^{\prime}}(|k| \leq 1)} \\
& \leq e^{-t / 2}\left\||k|^{m+\left(1 / q^{\prime}-1 / r^{\prime}\right)(3+\varepsilon)}\left[\widehat{\rho}_{ \pm 0}\right]\right\|_{L^{p^{\prime}}(|k| \leq 1)} \\
& \leq C e^{-t / 2}\left\|\nabla^{m+[3(1 / r-1 / q)]_{+}}\left[\hat{\rho}_{ \pm 0}\right]\right\|_{L^{r}} .
\end{aligned}
$$


To estimate the second part of the third term, we have

$$
\begin{aligned}
& \left\||k|^{m} \frac{1}{|k|} e^{-\theta t /|k|^{2}}\left[\widehat{\rho}_{ \pm 0}, \widehat{u}_{ \pm 0}, \widehat{E}_{0}, \widehat{B}_{0}\right]\right\|_{L^{q^{\prime}}}=\left(\left.\left.\int_{\mathbb{R}^{3}}|| k\right|^{m-1} e^{-\theta t /|k|^{2}}\left[\widehat{u}_{ \pm 0}, \widehat{E}_{0}, \widehat{B}_{0}\right]\right|^{q^{\prime}}\right)^{1 / q^{\prime}} \\
& =\left(\left.\left.\int_{\mathbb{R}^{3}}|| k\right|^{-\left(r^{\prime}-q^{\prime} / r^{\prime} q^{\prime}\right)(3+\epsilon)}|k|^{m+\left(r^{\prime}-q^{\prime} \mid r^{\prime} q^{\prime}\right)(3+\epsilon)} e^{-\theta t /|k|^{2}}\left[\widehat{u}_{ \pm 0}, \widehat{E}_{0}, \widehat{B}_{0}\right]\right|^{q^{\prime}}\right)^{1 / q^{\prime}} \\
& \leq\left\||k|^{-(3+\epsilon)}\right\|^{-\left(r^{\prime}-q^{\prime} / r^{\prime} q^{\prime}\right)}\left\||k|^{m+\left(r^{\prime}-q^{\prime} / r^{\prime} q^{\prime}\right)(3+\epsilon)} e^{-\theta t /|k|^{2}}\left[\widehat{u}_{ \pm 0}, \widehat{E}_{0}, \widehat{B}_{0}\right]\right\|_{L^{r^{\prime}}(|k| \leq 1)} \\
& \leq e^{-\theta t /|k|^{2}}\left\||k|^{m+l+3\left(1 / q^{\prime}-1 / r^{\prime}\right)}|k|^{-(l+1)}\left[u_{ \pm 0}, E_{0}, B_{0}\right]\right\|_{L^{r^{\prime}}(|k| \leq 1)} \\
& \leq C(1+t)^{-(l+1) / 2}\left\|\nabla^{m+[l+3(1 / r-1 / q)]_{+}}\left[u_{ \pm 0}, E_{0}, B_{0}\right]\right\|_{L^{r}} \text {, }
\end{aligned}
$$

where we used

$$
\sup _{|k| \leq 1} \frac{1}{|k|^{l+1}} e^{-\theta(t+1) /|k|^{2}} \leq C(1+t)^{-(l+1) / 2} .
$$

The proofs of (136) and (137) are similar.

Note that we transformed to the Fourier space to find the solution representation. Now, we go back to the physical space $x(t)$ and obtain the decay rates for the solutions to the linearized system. Fundamental properties of the system are given by the following corollary based on Theorem 5 .

Corollary 1. Assume $U(t)=e^{t L} U_{0}$ is the solution of the initial value problems (51) and (52) with initial data $\left[\rho_{ \pm 0}, u_{ \pm 0}, E_{0}, B_{0}\right]$ which satisfies (53). Then, $U=\left[\rho_{ \pm}, u_{ \pm}, E, B\right]$ satisfies

$$
\begin{aligned}
& \left\{\begin{array}{l}
\left\|\rho_{ \pm}(t)\right\| \leq C e^{-t / 2}\left\|\left[\rho_{ \pm 0}, u_{ \pm 0}\right]\right\| \\
\left\|u_{ \pm}(t)\right\| \leq C e^{-t / 2}\left\|\left[\rho_{ \pm 0}\right]\right\|+C(1+t)^{-5 / 4}\left\|\left[u_{ \pm 0}, E_{0}, B_{0}\right]\right\|_{L^{1} \cap \dot{H}^{2}}, \\
\|E(t)\| \leq C(1+t)^{-5 / 2}\left\|\left[u_{ \pm 0}, E_{0}, B_{0}\right]\right\|_{L^{1} \cap \dot{H}^{3}} \\
\|B(t)\| \leq C(1+t)^{-3 / 4}\left\|\left[u_{ \pm 0}, E_{0}, B_{0}\right]\right\|_{L^{1} \cap \dot{H}^{2}}
\end{array}\right. \\
& \left\{\begin{array}{l}
\left\|\rho_{ \pm}(t)\right\|_{L^{\infty}} \leq C e^{-t / 2}\left\|\left[\rho_{ \pm 0}, u_{ \pm 0}\right]\right\|_{L^{2} \cap \dot{H}^{2}} \\
\left\|u_{ \pm}(t)\right\|_{L^{\infty}} \leq C e^{-t / 2}\left\|\left[\rho_{ \pm 0}\right]\right\|_{L^{1} \cap \dot{H}^{2}}+C(1+t)^{-2}\left\|\left[u_{ \pm 0}, E_{0}, B_{0}\right]\right\|_{L^{1} \cap \dot{H}^{5}} \\
\|E(t)\|_{L^{\infty}} \leq C(1+t)^{-2}\left\|\left[u_{ \pm 0}, E_{0}, B_{0}\right]\right\|_{L^{1} \cap \dot{H}^{6}}, \\
\|B(t)\|_{L^{\infty}} \leq C(1+t)^{-3 / 2}\left\|\left[u_{ \pm 0}, E_{0}, B_{0}\right]\right\|_{L^{1} \cap \dot{H}^{5}}
\end{array}\right. \\
& \left\{\begin{array}{l}
\|B(t)\| \leq C(1+t)^{-5 / 4}\left\|\left[u_{ \pm 0}, E_{0}, B_{0}\right]\right\|_{L^{1} \cap \dot{H}^{4}}, \\
\left\|\nabla^{N}[E(t), B(t)]\right\| \leq C(1+t)^{-5 / 4}\left\|\left[u_{ \pm 0}, E_{0}, B_{0}\right]\right\|_{L^{1} \cap \dot{H}^{N+3}} .
\end{array}\right.
\end{aligned}
$$

Proof. The results of Corollary 1 are particular cases of Theorem 5.

For example, we explain how we get the decay rates for $u_{ \pm}$. From (135) for $L^{2}$, take $q=2, p=1, m=0, l=3 / 2$, and $r=2$ :

$$
\begin{aligned}
\left\|\nabla^{m} u_{ \pm}(t)\right\|_{L^{2}} \leq & C e^{-t / 2}\left(\left\|\rho_{ \pm 0}\right\|_{L^{1}}+\left\|\nabla^{[3 / 2]_{+1}}\left[\rho_{ \pm 0}, u_{ \pm 0}\right]\right\|_{L^{1}}\right) \\
& +C(1+t)^{-5 / 4}\left\|\left[u_{ \pm 0}, E_{0}, B_{0}\right]\right\|_{L^{1}} \\
& +C(1+t)^{-5 / 4}\left\|\nabla^{[1+1 / 2]_{+1}}\left[u_{ \pm 0}, E_{0}, B_{0}\right]\right\|_{L^{1}} .
\end{aligned}
$$

$$
\left\|u_{ \pm}(t)\right\|_{L^{2}} \leq C e^{-t / 2}\left\|\left[\rho_{ \pm 0}\right]\right\|+C(1+t)^{-5 / 4}\left\|\left[u_{ \pm 0}, E_{0}, B_{0}\right]\right\|_{L^{1} \cap \dot{H}^{2}}
$$

Now, for $L^{\infty}$, take $q=\infty, p=1, m=0, l=3$, and $r=2$ :

$$
\begin{aligned}
\left\|\nabla^{m} u_{ \pm}(t)\right\|_{L^{\infty}} \leq & C e^{-t / 2}\left(\left\|\rho_{ \pm 0}\right\|_{L^{1}}+\left\|\nabla^{[3 / 2]_{+1}}\left[\rho_{ \pm 0}, u_{ \pm 0}\right]\right\|_{L^{2}}\right) \\
& +C(1+t)^{-2}\left\|\left[u_{ \pm 0}, E_{0}, B_{0}\right]\right\|_{L^{1}} \\
& +C(1+t)^{-2}\left\|\nabla^{[4+1 / 2]_{+1}}\left[u_{ \pm 0}, E_{0}, B_{0}\right]\right\|_{L^{2}}
\end{aligned}
$$

Thus,

Thus, 
$\left\|u_{ \pm}(t)\right\|_{L^{\infty}} \leq C e^{-t / 2}\left\|\left[\rho_{ \pm 0}\right]\right\|_{L^{1} \cap \dot{H}^{2}}+C(1+t)^{-2}\left\|\left[u_{ \pm 0}, E_{0}, B_{0}\right]\right\|_{L^{1} \cap \dot{H}^{5}}$.

\section{Decay Rates for Nonlinear Systems}

4.1. Decay Rates for System (15). In this section, we apply the linear $L^{p}-L^{q}$ time decay property of the homogeneous system (51) to the nonlinear case. Throughout this section, we suppose that $U=\left[\rho_{ \pm}, u_{ \pm}, E, B\right]$ is the solution to the Cauchy problem (15) satisfying (16).

By Duhamel's principle, the solution $U$ can be formally written as

$$
U=e^{t L} U_{0}+\int_{0}^{t} e^{(t-s) L}\left[g_{1}(s), g_{2}(s), g_{3}(s), 0\right] \mathrm{d} s,
$$

where $e^{t L} U_{0}$ is as defined in Section 3.2 and

$$
\left\{\begin{aligned}
g_{1}= & -\nabla \cdot\left(\rho_{ \pm} u_{ \pm}\right), \\
g_{2}= & -u_{ \pm} \cdot \nabla u_{ \pm}-A_{ \pm} \gamma\left[\left(\rho_{ \pm}+1\right)^{\gamma-2}-1\right] \nabla \rho_{ \pm} \pm\left(u_{ \pm} \times B\right) \\
& +\alpha_{ \pm}\left(u_{ \pm}-u_{\mp}\right), \\
g_{3}= & \rho_{-} u_{-}-\rho_{+} u_{+} .
\end{aligned}\right.
$$

Remark 3. Note that in (156), $\left[g_{1}(s), g_{2}(s), g_{3}(s), 0\right]$ satisfies the compatibility condition (53). Thus, $e^{(t-s) L}$ acts on $\left[g_{1}(s), g_{2}(s), g_{3}(s), 0\right]$ for all $0 \leq s \leq t$.

Proposition 2. Suppose initial data $V_{0}=\left[\sigma_{ \pm 0}, v_{ \pm 0}, \widetilde{E_{0}}, \widetilde{B_{0}}\right]$ satisfies (17). If $\varepsilon_{N+2}\left(V_{0}\right)>0$ is small enough, then the solution $V=\left[\sigma_{ \pm}, v_{ \pm}, \widetilde{E}, \widetilde{B}\right]$ satisfies

$$
\|V(t)\|_{N} \leq C_{\varepsilon_{N+2}}\left(V_{0}\right)(1+t)^{-3 / 4},
$$

for any $t>0$. Moreover, $\varepsilon_{N+6}\left(V_{0}\right)>0$ being sufficiently small implies that the solution $V=\left[\sigma_{ \pm}, v_{ \pm}, \widetilde{E}, \widetilde{B}\right]$ satisfies

$$
\|\nabla V(t)\|_{N-1} \leq C_{\varepsilon_{N+6}}\left(V_{0}\right)(1+t)^{-5 / 4},
$$

for any $t \geq 0$.

Lemma 2. Suppose $V=\left[\sigma_{ \pm}, v_{ \pm}, E, B\right]$ is the solution of the initial value problems (15) and (16) with initial data $V_{0}=$ $\left[\sigma_{ \pm}, v_{ \pm}, \widetilde{E_{0}}, \widetilde{B_{0}}\right]$ satisfying (17) as described in Proposition 1 . If $\mathscr{E}_{N}\left(V_{0}\right)$ is sufficiently small, then

$$
\frac{\mathrm{d}}{\mathrm{d} t} \mathscr{E}_{N}(V(t))+\lambda \mathscr{D}_{N}(V(t)) \leq 0 .
$$

Proof. Let $l \geqslant 0$. Multiplying equation $(160)$ by $(1+t)^{l}$, we get

$$
\frac{\mathrm{d}}{\mathrm{d} t} \mathscr{E}_{N}(V(t))(1+t)^{l}+\lambda \mathscr{D}_{N}(V(t))(1+t)^{l} \leq 0 .
$$

Integrating over $[0,1]$, we find

$$
\int_{0}^{t} \frac{\mathrm{d}}{\mathrm{d} t} \mathscr{E}_{N}(V(s))(1+s)^{l} \mathrm{~d} s+\lambda \int_{0}^{t}(1+s)^{l} \mathscr{D}_{N}(V(s)) \mathrm{d} s \leq 0,
$$

which implies

$$
\begin{gathered}
(1+t)^{l} \mathscr{E}_{N}(V(t))+\lambda \int_{0}^{t}(1+s)^{l} \mathscr{D}_{N}(V(s)) \mathrm{d} s \\
\leq \mathscr{E}_{N}\left(V_{0}\right)+l \int_{0}^{t}(1+s)^{l-1} \mathscr{E}_{N}(V(s)) \mathrm{d} s .
\end{gathered}
$$

Recalling

$\mathscr{E}_{N}(V(t)) \leq C\left(\mathscr{D}_{N}(V(t))+\|\widetilde{B}\|^{2}+\left\|\sigma_{+}+\sigma_{-}\right\|^{2}\right)$,

we obtain

$$
\begin{aligned}
(1+t)^{l} \mathscr{E}_{N}(V(t))+\lambda \int_{0}^{t}(1+s)^{l} \mathscr{D}_{N}(V(s)) \mathrm{d} s \\
\leq \mathscr{E}_{N}\left(V_{0}\right)+C l \int_{0}^{t}(1+s)^{l-1}\left(\mathscr{D}_{N}(V(t))+\|\widetilde{B}\|^{2}+\left\|\sigma_{+}+\sigma_{-}\right\|^{2}\right) \mathrm{d} s \\
\quad+C l \int_{0}^{t}(1+s)^{l-1} \mathscr{D}_{N+1}(V(s)) \mathrm{d} s .
\end{aligned}
$$

Similarly,

$$
\begin{aligned}
& (1+t)^{l-1} \mathscr{E}_{N+1}(V(t))+\lambda \int_{0}^{t}(1+s)^{l-1} \mathscr{D}_{N+1}(V(s)) \mathrm{d} s \\
& \leq \mathscr{E}_{N+1}\left(V_{0}\right)+C l-1 \int_{0}^{t}(1+s)^{l-2} \\
& \cdot\left(\mathscr{D}_{N}(V(t))+\|\widetilde{B}\|^{2}+\left\|\sigma_{+}+\sigma_{-}\right\|^{2}\right) \mathrm{d} s \\
& \quad+C l+1 \int_{0}^{t}(1+s)^{l-2} \mathscr{D}_{N+2}(V(s)) \mathrm{d} s, \\
& \lambda \int_{0}^{t} \mathscr{D}_{N+2}(V(s)) \mathrm{d} s \leq \mathscr{E}_{N+2}\left(V_{t}\right)+\lambda \int_{0}^{t} \mathscr{D}_{N+2}(V(s)) \mathrm{d} s \\
& \leq \mathscr{E}_{N+2}\left(V_{0}\right),
\end{aligned}
$$

for $1 \leq l \leq 2$. Thus,

$$
\begin{aligned}
& (1+t)^{l} \mathscr{E}_{N}(V(t))+\lambda \int_{0}^{t}(1+s)^{l} \mathscr{D}_{N}(V(s)) \mathrm{d} s \\
& \quad \leq \mathscr{E}_{N}\left(V_{0}\right)+C \int_{0}^{t}(1+s)^{l-1}\left(\|\widetilde{B}\|^{2}+\left\|\sigma_{+}+\sigma_{-}\right\|^{2}\right) .
\end{aligned}
$$

To estimate the term on the right-hand side of (167), define

$$
\mathscr{E}_{N, \infty} \leq(1+s)^{3 / 2} \mathscr{E}_{N}(V(s))
$$

Applying Duhamel's principle for $t \geq 0$ along with the linear estimate on $B$ from (149) to (156), we get

$$
\begin{aligned}
\|B(t)\| \leq & (1+t)^{-3 / 4}\left\|\left[u_{ \pm 0}, E_{0}, B_{0}\right]\right\|_{L^{1} \cap \dot{H}^{2}} \\
& +C \int_{0}^{t}(1+s)^{-3 / 4}\left\|\left[g_{2}(s), g_{3}(s)\right]\right\|_{L^{1} \cap \dot{H}^{2}},
\end{aligned}
$$

for all $0 \leq s \leq t$.

Since 


$$
\begin{aligned}
\left\|g_{2}(s)\right\|_{L^{1}}= & \int \mid-u_{ \pm} \cdot \nabla u_{ \pm}-A_{ \pm} \gamma\left[\left(\rho_{ \pm}+1\right)^{\gamma-2}-1\right] \nabla \rho_{ \pm} \\
& \pm\left(u_{ \pm} \times B\right)+\alpha_{ \pm}\left(u_{ \pm}-u_{\mp}\right) \mid \mathrm{d} s \\
\leq & \int\left|-u_{ \pm} \cdot \nabla u_{ \pm}\right| \mathrm{d} s+\int\left|-u_{ \pm} \times B\right| \mathrm{d} s \\
& +\int\left|\gamma\left[\left(1+\rho_{ \pm}\right)^{\gamma-2}-1\right] \nabla \rho_{ \pm}\right| \mathrm{d} s
\end{aligned}
$$

we can apply the Sobolev imbedding theorem along with the Hölder and Cauchy inequalities to obtain

$$
\begin{gathered}
\left\|g_{2}(s)\right\|_{L^{1}} \leq C\left(\left\|\nabla u_{ \pm}\right\|+\left\|u_{ \pm}\right\|+\|B\|+\left\|\nabla \rho_{ \pm}\right\|\right), \\
\left\|g_{3}(s)\right\|_{L^{1}}=\int\left|\rho_{ \pm} u_{ \pm}\right| \mathrm{d} s .
\end{gathered}
$$

Thus,

$$
\left\|g_{3}(s)\right\|_{L^{1}} \leq C\left(\left\|\rho_{ \pm}\right\|+\left\|u_{ \pm}\right\|\right) .
$$

Therefore, we get

$$
\begin{aligned}
\left\|g_{2}(t)\right\|_{\dot{H}^{2}}= & \left(\sum_{\alpha=1}^{2} \mid D^{\alpha}\left(-u_{ \pm} \cdot \nabla u_{ \pm}-A_{ \pm} \gamma\right)\left[\left(\rho_{ \pm}+1\right)^{\gamma-2}-1\right] \nabla \rho_{ \pm}\right. \\
& \left. \pm\left(u_{ \pm} \times B\right)+\alpha_{ \pm}\left(u_{ \pm}-u_{\mp}\right) \mid\right) .
\end{aligned}
$$

Thus,

$$
\begin{aligned}
& \left\|g_{2}(t)\right\|_{\dot{H}^{2}} \leq\|\nabla U(t)\|_{2}\left\|u_{ \pm}, B(t)\right\|_{L^{\infty}}, \\
& \left\|g_{3}(s)\right\|_{\dot{H}^{2}}=\left(\sum_{\alpha=1}^{2} \int\left|D^{\alpha}\left(\rho_{ \pm} u_{ \pm}\right)\right|^{2} \mathrm{~d} s\right)^{1 / 2} .
\end{aligned}
$$

$$
\left\|g_{3}(t)\right\|_{\dot{H}^{2}} \leq\|\nabla U(t)\|_{2}\left\|\left[\rho_{ \pm}, u_{ \pm}\right]\right\|_{L^{\infty}} .
$$

Therefore,

$$
\left\|\left[g_{2}(s), g_{3}(s)\right]\right\|_{L^{1} \cap \dot{H}^{2}} \leq C \mathscr{E}_{N}(U(s)) .
$$

Notice that

$$
\mathscr{E}_{N}(U(s)) \leq C \mathscr{E}_{N}(V(\sqrt{\gamma} s)) .
$$

From (168),

$\mathscr{E}_{N}(V(\sqrt{\gamma} s)) \leq(1+\sqrt{\gamma} s)^{-3 / 2} \mathscr{E}_{N, \infty}(V(\sqrt{\gamma} t))$,

for any $0 \leq s \leq t$.

Then,

$\left\|\left[g_{2}(s), g_{3}(s)\right]\right\|_{L^{1} \cap \dot{H}^{2}} \leq C(1+\sqrt{\gamma} s)^{-3 / 2} \mathscr{C}_{N, \infty}(V(\sqrt{\gamma} t))$.

Substituting (180) in (169), we get

$$
\begin{aligned}
\|B(t)\| \leq & (1+t)^{-3 / 4}\left(\left\|\left[u_{ \pm 0}, E_{0}, B_{0}\right]\right\|_{L^{1} \cap \dot{H}^{2}}\right. \\
& \left.+C(1+s)^{-3 / 2} \mathscr{E}_{N, \infty}(V(\sqrt{\gamma} t))\right) \\
\leq & (1+t)^{-3 / 4}\left(\left\|\left[u_{ \pm 0}, E_{0}, B_{0}\right]\right\|_{L^{1} \cap \dot{H}^{2}}+\mathscr{E}_{N, \infty}(V(\sqrt{\gamma} t))\right),
\end{aligned}
$$

which implies

$$
\|\widetilde{B}(t)\|^{2} \leq(1+t)^{-3 / 2}\left(\left\|\left[v_{ \pm 0}, \widetilde{E}_{0}, \widetilde{B}_{0}\right]\right\|_{L^{1} \cap \dot{H}^{2}}^{2}+\left(\mathscr{E}_{N, \infty}(V(\sqrt{\gamma} t))\right)^{2}\right) .
$$

Finally, we need to show the uniform-in-time bound of $\mathscr{E}_{N, \infty}(V(t))$ which implies the decay rates of the energy functional $\mathscr{E}_{N}(V(t))$. Using $l=3 / 2+\epsilon$ in (172) with $\epsilon>0$ sufficiently small and using (181), we get

Thus,

$$
\begin{aligned}
(1 & +t)^{(3 / 2)+\epsilon} \mathscr{E}_{N}(V(t))+\lambda \int_{0}^{t}(1+s)^{(3 / 2)+\epsilon} \mathscr{D}_{N}(V(s)) \mathrm{d} s \\
& \leq C \mathscr{E}_{N+2}\left(V_{0}\right)+C \int_{0}^{t}(1+s)^{1 / 2+\epsilon}\left(\|\widetilde{B}\|^{2}+\left\|\sigma_{+}+\sigma_{-}\right\|^{2}\right) \\
& \leq C \mathscr{E}_{N+1}\left(V_{0}\right)+(1+t)^{(3 / 2)+\epsilon}\left(C(1+t)^{-3 / 2}\left\|\left[v_{ \pm 0}, \widetilde{E}_{0}, \widetilde{B}_{0}\right]\right\|_{L^{1} \cap \dot{H}^{2}}^{2} C(1+s)^{-3 / 2}\left(\mathscr{E}_{N, \infty}(V(t))\right)^{2}\right) \\
& \leq C \mathscr{E}_{N+2}\left(V_{0}\right)+C(1+t)^{\epsilon}\left(\left\|\left[v_{ \pm 0}, \widetilde{E}_{0}, \widetilde{B}_{0}\right]\right\|_{L^{1} \cap \dot{H}^{2}}^{2}\left(\mathscr{E}_{N, \infty}(V(t))\right)^{2}\right),
\end{aligned}
$$

which implies

$$
\begin{aligned}
(1+t)^{3 / 2} \mathscr{E}_{N}(V(t)) \leq & C\left(\mathscr{E}_{N+2}\left(V_{0}\right)+\left\|\left[v_{ \pm 0}, \widetilde{E}_{0}, \widetilde{B}_{0}\right]\right\|_{L^{1}}^{2}\right. \\
& \left.+\left(\mathscr{E}_{N, \infty}(V(t))\right)^{2}\right), \\
(1+t)^{3 / 2}\left(\mathscr{E}_{N, \infty}(V(t))\right) \leq & C\left(\epsilon_{N+2}\left(V_{0}\right)^{2}+\left(\mathscr{E}_{N, \infty}(V(t))\right)^{2}\right) .
\end{aligned}
$$

Now, from the definition (28) and since $\epsilon_{N+2}\left(V_{0}\right)>0$ is sufficiently small, $\mathscr{E}_{N, \infty}(V(t)) \leq \epsilon_{N+2}\left(V_{0}\right)^{2}$ holds for any $t \geq 0$, and we get

$$
\|V(t)\|_{N} \leq C\left(\mathscr{E}_{N}(V(t))\right)^{1 / 2} \leq C \epsilon_{N+2}\left(V_{0}\right)(1+t)^{-3 / 4},
$$

which is the proof of (158) in Proposition 2. 
4.2. Time Rate for the High-Order Energy Functional. In this section, we determine the time decay estimates of the highorder energy functional $\|\nabla V\|_{N}^{2}$, that is, the proof (159) of Proposition 2. To do so, we investigate the time decay estimates on $\|\nabla \widetilde{B}\|$ and $\left\|\nabla^{N}[\widetilde{E}, \widetilde{B}]\right\|$ using the following lemma.

Lemma 3. Suppose $V=\left[\sigma_{ \pm}, v_{+}, \widetilde{E}, \widetilde{B}\right]$ is the solution to the Cauchy problems (15) and (16) with initial condition $V_{0}=$ $\left[\sigma_{0}, v_{0}, \widetilde{E}_{0}, \widetilde{B}_{0}\right]$ satisfying (17) as obtained in Proposition 1. If $\mathscr{E}_{N}\left(V_{0}\right)$ is sufficiently small, then there exists the high-order energy functional $\mathscr{E}_{N}^{h}(\cdot)$ and the high-order dissipation rate $\mathscr{D}_{N}^{h}(\cdot)$ such that for all $\geq 0$,

$$
\left.\left.\frac{\mathrm{d}}{\mathrm{d} t} \mathscr{E}_{N}^{h}(V(t))\right)+\lambda \mathscr{D}_{N}^{h}(V(t))\right) \leq C\|\nabla \widetilde{B}\|^{2} .
$$

By comparing the definitions of $\mathscr{E}_{N}^{h}(V(t))$ (22) and $\mathscr{D}_{N}^{h}(V(t))(25)$ and based on Lemma 3 equation (186), we have

$$
\begin{aligned}
& \frac{\mathrm{d}}{\mathrm{d} t} \mathscr{E}_{N}^{h}(V(t))+\lambda \mathscr{E}_{N}^{h}(V(t)) \leq C\left(\|\nabla \widetilde{B}\|^{2}+\left\|\nabla^{N}[\widetilde{E}, \widetilde{B}]\right\|\right. \\
& \left.\quad+\left\|\nabla\left(\sigma_{+}+\sigma_{-}\right)\right\|^{2}\right),
\end{aligned}
$$

which implies

$$
\begin{aligned}
\mathscr{E}_{N}^{h}(V(t)) \leq & e^{-\lambda t} \mathscr{E}_{N}^{h}\left(V_{0}\right)+C \int_{0}^{t} e^{-\lambda(t-s)}\left(\|\nabla \widetilde{B}(s)\|^{2}\right. \\
& \left.+\left\|\nabla^{N}[\widetilde{E}, \widetilde{B}](s)\right\|+\left\|\nabla\left(\sigma_{+}+\sigma_{-}\right)(s)\right\|^{2}\right) .
\end{aligned}
$$

Now, to estimate the time integral term on the righthand side of inequality (189), we have the next lemma.

Lemma 4. Suppose $V=\left[\sigma_{ \pm}, v_{ \pm}, \widetilde{E}, \widetilde{B}\right]$ is the solution of the initial value problems (15) and (16) with initial condition $V_{0}=\left[\sigma_{0}, v_{0}, \widetilde{E_{0}}, \widetilde{B_{0}}\right]$ satisfying (17) as obtained in Proposition 1. If $\left.\epsilon_{N+6}\left(V_{0}\right)\right)$ is sufficiently small, where defined in (28), then for all $t \geq 0$,

$$
\begin{aligned}
& \|\nabla \widetilde{B}(t)\|^{2}+\left\|\nabla^{N}[\widetilde{E}(t), \widetilde{B}(t)]\right\|^{2}+\left\|\nabla^{N}\left(\sigma_{+}(t)+\sigma_{-}(t)\right)\right\|^{2} \\
& \quad \leq \varepsilon_{N+6}\left(V_{0}\right)^{2}(1+t)^{5 / 2} .
\end{aligned}
$$

The proofs of Lemmas 3 and 4 are analogous to those of Lemmas 5.1 and 5.2 in [10].

Now, we suppose that the above lemma is true. Then, by putting (188) into (186), we get

$$
\mathscr{E}_{N}^{h}(V(t)) \leq e^{-\lambda t} \mathscr{E}_{N}^{h}\left(V_{0}\right)+C \epsilon_{N+6}\left(V_{0}\right)^{2}(1+t)^{5 / 2},
$$

in which (159) in Proposition 2 holds.

4.3. Decay Rate in $L^{q}$. In this section, we determine the decay rates in $L^{q}, 2 \leq q \leq+\infty$, of (7)-(9) for solutions $U=\left[\rho_{ \pm}\right.$, $\left.u_{ \pm}, E, B\right]$ to the Cauchy problems (51) and (52. Suppose that
$\epsilon_{13}\left(V_{0}\right)$ is sufficiently small. In addition, for $N \geq 4$, Proposition 2 shows that if $\epsilon_{N+2}\left(V_{0}\right)$ is sufficiently small, then

$$
\|U(t)\|_{N} \leq C \epsilon_{N+2}\left(V_{0}\right)(1+t)^{-3 / 4},
$$

and if $\epsilon_{N+6}\left(V_{0}\right)$ is sufficiently small, then

$$
\|\nabla U(t)\|_{N-1} \leq C \epsilon_{N+6}\left(V_{0}\right)(1+t)^{-5 / 4} \text {. }
$$

We establish the estimates on $B,\left[u_{+}, E\right]$ and $\rho_{+}$as follows.

To estimate the $L^{2}$ decay rate on $\|B\|_{L^{q}}$, note that from (191), we obtain

$$
\|B(t)\| \leq C \epsilon_{6}\left(V_{0}\right)(1+t)^{-3 / 4} .
$$

To estimate the $L^{\infty}$ decay rate, note that the $L^{\infty}$ estimates on $B$ in (150) to (156) give

$$
\begin{aligned}
\|B(t)\|_{L^{\infty}} \leq & C(1+t)^{-3 / 2}\left\|\left[u_{ \pm 0}, E_{0}, B_{0}\right]\right\|_{L^{1} \cap \dot{H}^{5}} \\
& +C \int_{0}^{t}(1+t-s)^{-3 / 2}\left\|\left[g_{2}(s), g_{3}(s)\right]\right\|_{L^{1} \cap \dot{H}^{5}} \mathrm{~d} s .
\end{aligned}
$$

From (191), we obtain

$$
\left\|\left[g_{2}(s), g_{3}(s)\right]\right\|_{L^{1} \cap \dot{H}^{5}} \leq C\|U(t)\|_{6}^{2} \leq C \epsilon_{8}^{2}\left(V_{0}\right)(1+t)^{-3 / 2} \text {, }
$$

and thus

$$
\|B(t)\|_{L^{\infty}} \leq C \epsilon_{8}\left(V_{0}\right)(1+t)^{-3 / 2} .
$$

Moreover, by $L^{2}-L^{\infty}$ interpolation,

$$
\|B(t)\|_{L^{q}} \leq C \epsilon_{8}\left(V_{0}\right)(1+t)^{-3 / 2+3 / 2 q},
$$

for $2 \leq q \leq \infty$

To estimate $\left\|u_{ \pm}, E\right\|_{L^{q}}$, note that for the $L^{2}$ decay rate, we can utilize the $L^{2}$ estimate on $u_{ \pm}$and $E$ in (149) to (156). That is,

$$
\begin{aligned}
\left\|u_{ \pm}(t)\right\| \leq & C(1+t)^{-\frac{5}{4}}\left(\left\|\rho_{ \pm 0}\right\|+\left\|\left[u_{ \pm 0}, E_{0}, B_{0}\right]\right\|_{L^{1} \cap \dot{H}^{2}}\right) \\
& +C \int_{0}^{t}(1+t-s)^{-5 / 4}\left(\left\|g_{1}(s)\right\|+\left\|\left[g_{2}(s), g_{3}(s)\right]\right\|_{L^{1} \cap \dot{H}^{2}}\right) \mathrm{d} s, \\
\|E(t)\| \leq & C(1+t)^{-5 / 4}\left\|\left[u_{ \pm 0}, E_{0}, B_{0}\right]\right\|_{L^{1} \cap \dot{H}^{3}} \\
& +C \int_{0}^{t}(1+t-s)^{-5 / 4}\left\|\left[g_{2}(s), g_{3}(s)\right]\right\|_{L^{1} \cap \dot{H}^{3}} \mathrm{~d} s .
\end{aligned}
$$

From (191),

$$
\left\|g_{1}(t)\right\|+\left\|\left[g_{2}(t), g_{3}(t)\right]\right\|_{L^{1} \cap \dot{H}^{3}} \leq\|U(t)\|_{4}^{2} \leq C \epsilon_{6}^{2}\left(V_{0}\right)(1+t)^{-3 / 2},
$$

and it therefore holds that

$$
\left\|u_{ \pm}(t), E(t)\right\| \leq C \epsilon_{6}\left(V_{0}\right)(1+t)^{-5 / 4} .
$$

For the $L^{\infty}$ decay rate, we can utilize the $L^{\infty}$ estimates on $u_{ \pm}$and $E$ in (150) to (156). That is, 


$$
\begin{aligned}
\left\|u_{ \pm}(t)\right\|_{L^{\infty}} \leq & C(1+t)^{-2}\left(\left\|\rho_{ \pm 0}\right\|_{L^{2} \cap \dot{H}^{2}}+\left\|\left[u_{ \pm 0}, E_{0}, B_{0}\right]\right\|_{L^{1} \cap \dot{H}^{5}}\right) \\
& +C \int_{0}^{t}(1+t-s)^{-2}\left(\left\|g_{1}(s)\right\|_{L^{2} \cap \dot{H}^{2}}\right. \\
& \left.+\left\|\left[g_{2}(s), g_{3}(s)\right]\right\|_{L^{1} \cap \dot{H}^{5}}\right) \mathrm{d} s, \\
\|E(t)\|_{L^{\infty}} \leq & C(1+t)^{-2}\left\|\left[u_{ \pm 0}, E_{0}, B_{0}\right]\right\|_{L^{1} \cap \dot{H}^{6}} \\
& +C \int_{0}^{t}(1+t-s)^{-2}\left\|\left[g_{2}(s), g_{3}(s)\right]\right\|_{L^{1} \cap \dot{H}^{6}} \mathrm{~d} s .
\end{aligned}
$$

Since

$$
\begin{aligned}
& \left\|g_{1}(t)\right\|_{L^{2} \cap \dot{H}^{2}}+\left\|\left[g_{2}(t), g_{3}(t)\right]\right\|_{\dot{H}^{5} \cap \dot{H}^{6}} \leq\|\nabla U(t)\|_{6}^{2} \\
& \quad \leq C \epsilon_{13}^{2}\left(V_{0}\right)(1+t)^{-5 / 2}, \\
& \left\|\left[g_{2}(t), g_{3}(t)\right]\right\|_{L^{1}} \leq C\|U(t)\|\left(\left\|u_{ \pm}(t)\right\|+\|\nabla U(t)\|\right) \\
& \quad \leq C\left[\epsilon_{6}\left(V_{0}\right)(1+t)^{-3 / 4}\right] \cdot\left[\epsilon_{10}\left(V_{0}\right)(1+t)^{-5 / 4}\right] \\
& \quad \leq C \epsilon_{10}^{2}\left(V_{0}\right)(1+t)^{-2},
\end{aligned}
$$

it holds that

$$
\left\|u_{ \pm}(t), E(t)\right\|_{L^{\infty}} \leq C \varepsilon_{13}\left(V_{0}\right)(1+t)^{-2} .
$$

Moreover, by $L^{2}-L^{\infty}$ interpolation,

$$
\left\|u_{ \pm}(t), E(t)\right\|_{L^{9}} \leq C \varepsilon_{13}\left(V_{0}\right)(1+t)^{-2+(3 / 2 q)},
$$

for $2 \leq q \leq \infty$.

To estimate the $L^{2}$ decay rate on $\left\|\rho_{ \pm}\right\|_{L^{q}}$, we utilize the $L^{2}$ estimate on $\rho_{ \pm}$in (149) to (156). We get

$$
\left\|\rho_{ \pm}(t)\right\| \leq C e^{-t / 2}\left\|\left[\rho_{ \pm 0}, u_{ \pm 0}\right]\right\|+C \int_{0}^{t} e^{-(t-s) / 2}\left\|\left[g_{1}(s), g_{2}(s)\right]\right\| \mathrm{d} s .
$$

Since

$$
\begin{aligned}
&\left\|\left[g_{1}(t), g_{2}(t)\right]\right\| \leq C\left(\|\nabla U(t)\|_{1}^{2}+\left\|u_{ \pm}(t)\right\| \cdot\|B(t)\|_{L^{\infty}}\right) \\
& \leq C \epsilon_{10}^{2}\left(V_{0}\right)(1+t)^{-5 / 2} \\
&\left\|\rho_{ \pm}(t)\right\| \leq C \epsilon_{10}\left(V_{0}\right)(1+t)^{-5 / 2} .
\end{aligned}
$$

(205) implies the slower decay estimate:

Moreover, after estimating $\left\|\left[g_{1}(t), g_{2}(t)\right]\right\|$ and utilizing the previous slower decay estimate, we obtain

$$
\begin{array}{r}
\left\|\left[g_{1}(t), g_{2}(t)\right]\right\| \leq C\left\|u_{ \pm}(t)\right\|_{L^{\infty}}\left(\left\|\nabla \rho_{ \pm}(t)\right\|+\left\|\nabla u_{ \pm}(t)\right\|+\|B(t)\|\right) \\
\leq C\left\|\rho_{ \pm}(t)\right\|\left(\left\|\nabla \rho_{ \pm}(t)\right\|_{2}+\left\|\nabla u_{ \pm}(t)\right\|_{2}\right) \leq C \epsilon_{13}\left(V_{0}\right)^{2}(1+t)^{-11 / 4},
\end{array}
$$

and it follows from (205) that

$$
\left\|\rho_{ \pm}(t)\right\| \leq C \epsilon_{13}\left(V_{0}\right)(1+t)^{-11 / 4} .
$$

For the $L^{\infty}$ decay rate, we utilize the $L^{\infty}$ estimates on $\rho_{ \pm}$ in (150) to (156). We have

$$
\begin{aligned}
\left\|\rho_{ \pm}(t)\right\|_{L^{\infty}} \leq & C e^{t / 2}\left\|\rho_{ \pm 0}, u_{ \pm}\right\|_{L^{2} \cap \dot{H}^{2}} \\
& +C \int_{0}^{t} e^{-(t-s) / 2}\left\|\left[g_{1}(s), g_{2}(s)\right]\right\|_{L^{2} \cap \dot{H}^{2}} \mathrm{~d} s
\end{aligned}
$$

and it is simple to check that

$$
\begin{aligned}
\left\|\left[g_{1}(t), g_{2}(t)\right]\right\|_{L^{2} \cap \dot{H}^{2}} \leq & C\|\nabla U(t)\|_{4}\left(\left\|\rho_{ \pm}(t)\right\|+\left\|u_{ \pm}(t), B(t)\right\|_{L^{\infty}}\right. \\
& \left.+\left\|\nabla\left[\rho_{ \pm}(t), u_{ \pm}(t)\right]\right\|_{L^{\infty}}\right) \\
\leq & C \epsilon_{13}\left(V_{0}\right)(1+t)^{-11 / 4} .
\end{aligned}
$$

Applying (210), we obtain

$$
\left\|\rho_{ \pm}(t)\right\|_{L^{\infty}} \leq C \epsilon_{13}\left(V_{0}\right)(1+t)^{-11 / 4} .
$$

Thus, by $L^{2}-L^{\infty}$ interpolation,

$$
\left\|\rho_{ \pm}(t)\right\|_{L^{q}} \leq C \epsilon_{13}\left(V_{0}\right)(1+t)^{-11 / 4},
$$

for $2 \leq q \leq \infty$. Note that (197), (204), and (213) correspond to (9), (8), and (7), respectively. This completes the proof of Theorem 1.

Now, to obtain the decay rates of smooth solutions from (156) let $G(t) * u_{ \pm 0}=e^{t L} U_{0}$. For the $L^{2}$ decay rate, by applying (156) and (157), we have

$$
\begin{aligned}
\left\|U-e^{t L} U_{0}\right\|^{2} & =\left\|\int_{0}^{t} e^{(t-s) L}\left[g_{1}(s), g_{2}(s), g_{3}(s), 0\right] \mathrm{d} s\right\|^{2} \\
& \leq \int_{0}^{t} e^{(t-s) L}\left\|\left[g_{1}(s), g_{2}(s), g_{3}(s), 0\right]\right\|^{2} \mathrm{~d} s,
\end{aligned}
$$

since

$$
\begin{aligned}
\left\|g_{1}(t)\right\|_{L^{2}}^{2}= & \left\|\int_{0}^{t} e^{(t-s) L}\left(-\nabla \cdot\left(\rho_{ \pm} u_{ \pm}\right)\right) d s\right\|^{2} \\
\leq & \int_{0}^{t} e^{(t-s) L}\left\|\nabla \cdot\left(\rho_{ \pm} u_{ \pm}\right)\right\|^{2} d s \\
\leq & C \epsilon_{10}^{2}\left(V_{0}\right)(1+t)^{-5 / 2}, \\
\left\|g_{2}(t)\right\|_{L^{2}}^{2}= & \| \int_{0}^{t} e^{(t-s) L}\left(-u_{ \pm} \cdot \nabla u_{ \pm}-A_{ \pm} \gamma\left[\left(\rho_{ \pm}+1\right)^{\gamma-2}-1\right] \nabla \rho_{ \pm}\right. \\
& \left. \pm\left(u_{ \pm} \times B\right)+\alpha_{ \pm}\left(u_{ \pm}-u_{\mp}\right)\right) \mathrm{d} s \|^{2} \\
\leq & \int_{0}^{t} e^{(t-s) L} \|-u_{ \pm} \cdot \nabla u_{ \pm}-A_{ \pm} \gamma\left[\left(\rho_{ \pm}+1\right)^{\gamma-2}-1\right] \nabla \rho_{ \pm} \\
& \pm\left(u_{ \pm} \times B\right)+\alpha_{ \pm}\left(u_{ \pm}-u_{\mp}\right) \|^{2} \mathrm{~d} s \\
\leq & C \epsilon_{10}^{2}\left(V_{0}\right)(1+t)^{-5 / 2}, \\
\left\|g_{3}(t)\right\|_{L^{2}}^{2}= & \left\|\int_{0}^{t} e^{(t-s) L}\left(\rho_{-} u_{-}-\rho_{+} u_{+}\right) \mathrm{d} s\right\|^{2} \\
\leq & \int_{0}^{t} e^{(t-s) L}\left\|\rho_{-} u_{-}-\rho_{+} u_{+}\right\|^{2} \mathrm{~d} s \\
\leq & C \epsilon_{10}^{2}\left(V_{0}\right)(1+t)^{-5 / 2} .
\end{aligned}
$$


Therefore,

$$
\left\|U-e^{t L} U_{0}\right\|_{L^{2}} \leq C \epsilon_{10}\left(V_{0}\right)(1+t)^{-5 / 2} .
$$

For the $L^{\infty}$ decay rate, by applying (156) and (157), we get

$$
\begin{aligned}
\left\|g_{1}(t)\right\|_{L^{\infty}}= & \sup _{0 \leq s \leq t}\left|\int_{0}^{t} e^{(t-s) L}\left(-\nabla \cdot\left(\rho_{ \pm} u_{ \pm}\right)\right) \mathrm{d} s\right| \\
\leq & \sup _{0 \leq s \leq t} \int_{0}^{t} e^{(t-s) L}\left|\nabla \cdot\left(\rho_{ \pm} u_{ \pm}\right)\right| \mathrm{d} s \\
\leq & C \epsilon_{13}\left(V_{0}\right)(1+t)^{-2}, \\
\left\|g_{2}(t)\right\|_{L^{\infty}}= & \sup _{0 \leq s \leq t} \mid \int_{0}^{t} e^{(t-s L)}\left(-u_{ \pm} \cdot \nabla u_{ \pm}-A_{ \pm} \gamma\left[\left(\rho_{ \pm}+1\right)^{\gamma-2}-1\right]\right. \\
& \left.\left.\nabla \rho_{ \pm} \pm\left(u_{ \pm} \times B\right)\right)+\alpha_{ \pm}\left(u_{ \pm}-u_{\mp}\right)\right) \mathrm{d} s \mid \\
\leq & \sup _{0 \leq s \leq t} \int_{0}^{t} e^{(t-s) L} \mid-u_{ \pm} \cdot \nabla u_{ \pm}-A_{ \pm} \gamma\left[\left(\rho_{ \pm}+1\right)^{\gamma-2}-1\right] \\
& \nabla \rho_{ \pm} \pm\left(u_{ \pm} \times B\right)+\alpha_{ \pm}\left(u_{ \pm}-u_{\mp}\right) \mid \mathrm{d} s \\
\leq & C \epsilon_{13}\left(V_{0}\right)(1+t)^{-2}, \\
= & \sup _{0 \leq s \leq t}\left|\int_{0}^{t} e^{(t-s) L}\left(\rho_{-} u_{-}-\rho_{+} u_{+}\right) d s\right| \\
\leq & \sup _{0 \leq s \leq t} \int_{0}^{t} e^{(t-s) L}\left|\rho_{-} u_{-}-\rho_{+} u_{+}\right| d s \\
\leq & C \epsilon_{13}\left(V_{0}\right)(1+t)^{-2} .
\end{aligned}
$$

Thus,

$$
\left\|U-e^{t L} U_{0}\right\|_{L^{\infty}} \leq C \epsilon_{13}\left(V_{0}\right)(1+t)^{-2} .
$$

So, by $L^{2}-L^{\infty}$ interpolation,

$$
\left\|U-e^{t L} U_{0}\right\|_{L^{q}} \leq C \epsilon_{13}\left(V_{0}\right)(1+t)^{-2+(3 / 2 q)},
$$

for $2 \leq q \leq \infty$. This completes the proof of Theorem 2 .

\section{Data Availability}

The data used to support the findings of this study are included within the article.

\section{Conflicts of Interest}

The authors declare that there are no conflicts of interest regarding the publication of this paper.

\section{References}

[1] G.-Q. Chen, J. W. Jerome, and D. Wang, "Compressible Euler-Maxwell equations," Transport Theory and Statistical Physics, vol. 29, no. 3-5, pp. 311-331, 2000.

[2] R. J. Duan, "The Cauchy problem on the compressible twofluids Euler-Maxwell equations," SIAM Journal on Mathematical Analysis, vol. 44, no. 1, pp. 102-133, 2012.

[3] Y.-J. Peng, S. Wang, and Q. Gu, "Relaxation limit and global existence of smooth solutions of compressible Euler-Maxwell equations," SIAM Journal on Mathematical Analysis, vol. 43, no. 2, pp. 944-970, 2011.
[4] Y. Ueda, S. Wang, and S. Kawashima, "Dissipative structure of the regularity-loss type and time asymptotic decay of solutions for the Euler-Maxwell system," SIAM Journal on Mathematical Analysis, vol. 44, no. 3, pp. 2002-2017, 2012.

[5] Y.-J. Peng and S. Wang, "Convergence of compressible Euler-Maxwell equations to incompressible Euler equations," Communications in Partial Differential Equations, vol. 33, no. 3, pp. 349-376, 2008.

[6] Y.-J. Peng and S. Wang, "Rigorous derivation of incompressible e-MHD equations from compressible Euler-Maxwell equations," SIAM Journal on Mathematical Analysis, vol. 40, no. 2, pp. 540-565, 2008.

[7] J. Yang, S. Wang, Y. Li, and D. Luo, "The diffusive relaxation limit of non-isentropic Euler-Maxwell equations for plasmas," Journal of Mathematical Analysis and Applications, vol. 380, no. 1, pp. 343-353, 2011.

[8] Y.-H. Feng, S. Wang, and S. Kawashima, "Global existence and asymptotic decay of solutions to the non-isentropic Euler-Maxwell system," Mathematical Models and Methods in Applied Sciences, vol. 24, no. 14, pp. 2851-2884, 2014.

[9] C. Besse, P. Degond, F. Deluzet, J. Claudel, G. Gallice, and C. Tessieras, "A model hierarchy for ionospheric plasma modeling," Mathematical Models and Methods in Applied Sciences, vol. 14, no. 3, pp. 393-415, 2004.

[10] R. Duan, "Global smooth flows for the compressible Euler-Maxwell system: the relaxation case," Journal of $\mathrm{Hy}$ perbolic Differential Equations, vol. 8, no. 2, pp. 375-413, 2011.

[11] S. Kawashima and Y. Ueda, "Decay property of regularity-loss type for the Euler-Maxwell system," Methods and Applications of Analysis, vol. 18, pp. 215-268, 2011.

[12] J. W. Jerome, "The Cauchy problem for compressible hydrodynamic-Maxwell systems: a local theory for smooth solutions," Differential Integral Equations, vol. 16, pp. 1345-1368, 2003.

[13] T. Kato, "The Cauchy problem for quasi-linear symmetric hyperbolic systems," Archive for Rational Mechanics and Analysis, vol. 58, no. 3, pp. 181-205, 1975.

[14] M. E. Taylor, Partial Differential Equations, III. Basic Theory, Springer, New York, NY, USA, 1996. 